\title{
Regional brain abnormalities in behavioral variant frontotemporal dementia: a meta-analysis
}

Aida Kamalian ${ }^{a *}$, Tina Khodadadifar ${ }^{\text {b*, }}$, Amin Saberic, Maryam Masoudia ${ }^{\mathrm{a}}$, Julia A. Camilleri ${ }^{\mathrm{d}, \mathrm{e}}$, Claudia R. Eickhoff ${ }^{\mathrm{d}, \mathrm{f}}$, Mojtaba Zarei ${ }^{\mathrm{c}}$, Lorenzo Pasquini ${ }^{\mathrm{g}}$, Simon B. Eickhoff ${ }^{\mathrm{d}, \mathrm{e}}$, Masoud Tahmasian ${ }^{\mathrm{c} * *}$

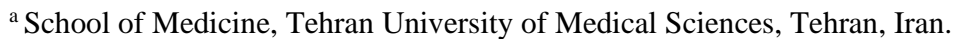

${ }^{\mathrm{b}}$ School of Cognitive Sciences, Institute for Research in Fundamental Sciences, Tehran, Iran.

${ }^{c}$ Institute of Medical Science and Technology, Shahid Beheshti University, Tehran, Iran.

${ }^{\mathrm{d}}$ Institute of Neuroscience and Medicine (INM-7), Research Center Jülich, Jülich, Germany.

${ }^{\mathrm{e}}$ Institute for Systems Neuroscience, Medical Faculty, Heinrich-Heine University Düsseldorf, Germany.

${ }^{\mathrm{f}}$ Institute of Clinical Neuroscience and Medical Psychology, Heinrich Heine University Düsseldorf, Düsseldorf, Germany.

${ }^{g}$ Department of Neurology, Memory and Aging Center, University of California, San Francisco, San Francisco, CA, USA.

* A.K. and T.K. contributed equally to this work.

**Corresponding Author: Masoud Tahmasian, Institute of Medical Science and Technology, Shahid Beheshti University, Daneshjou Boulevard, Velenjak, P.O. Box 1983969411, Tehran, Iran; Tel: +982129905803; masoudtahmasian@gmail.com

Declarations of interest: none

Running title: Neuroimaging Meta-analysis in bvFTD 


\begin{abstract}
INTRODUCTION: Numerous studies have reported brain alterations in behavioral variant frontotemporal dementia (bvFTD). However, they pointed to inconsistent findings.

METHODS: We used a meta-analytic approach to identify the convergent structural and functional brain abnormalities in bvFTD. Following the best-practice neuroimaging metaanalysis guidelines, we searched PubMed and Embase databases and performed reference tracking. Then, the coordinates of group comparisons between bvFTD and controls from 73 studies were extracted and tested for convergence using activation likelihood estimation.

RESULTS: We identified convergent abnormalities in the anterior cingulate cortices, anterior insula, amygdala, paracingulate, striatum, and hippocampus. Task-based and resting-state functional connectivity pointed to the joint networks that are connected to the obtained consistent regions. Functional decoding analyses suggested associated dysfunction of emotional processing, interoception, reward processing, higher-order cognitive functions, olfactory and gustatory perceptions in bvFTD.
\end{abstract}

DISCUSSION: Our findings highlighted a key role of the salience network and subcortical regions in the pathophysiology of bvFTD.

Keywords: Behavioral Variant Frontotemporal Dementia; Activation Likelihood Estimation; Voxel-Based Morphometry; Positron Emission Tomography; Functional Magnetic Resonance Imaging. 


\section{Introduction}

Behavioral-variant frontotemporal dementia (bvFTD) is a neurodegenerative syndrome characterized by neurodegeneration in the frontal and anterior temporal lobes leading to insidious and progressive changes in behavior, personality and social functions [1]. BvFTD is the most common frontotemporal dementia (FTD) syndrome and the second major cause of early-onset dementia after Alzheimer's disease (AD). The prevalence of bvFTD is estimated to be approximately 13/100,000 with at sixties which is the peak age of onset of bvFTD [1]. Given the heterogeneous symptomology and gradual course of bvFTD, early detection of bvFTD is often abstruse and causes frustrating experiences for patients and relatives [2]. Around $50 \%$ of patients are initially misdiagnosed with psychiatric disorders due to the overlapping behavioral symptoms and in later stages with $\mathrm{AD}$ due to cognitive impairments. Recently, diagnostic accuracy of bvFTD has been improved dramatically by introducing the consensus clinical criteria that rely on a hybrid approach incorporating clinical symptoms and neuropsychological assessment for "possible bvFTD", additional neuroimaging testing and cognitive decline evaluation for "probable bvFTD", and further neuropathology and genetic confirmation for "definite bvFTD" [3]. Thus, nowadays, neuroimaging is an essential step for bvFTD diagnosis.

Numerous neuroimaging studies identified structural and functional brain alterations in patients with bvFTD. In particular, structural magnetic resonance imaging (MRI) techniques including Voxel-Based Morphometry (VBM) have provided collective evidence for regional brain atrophy predominantly in the frontal and anterior temporal lobes, insula, anterior cingulate and several subcortical structures including the amygdala, hippocampus, striatum, putamen, thalamus, caudate and hypothalamus [4]. As such, presence of pathological atrophy in the frontotemporal regions is one of the diagnostic criteria of "probable" bvFTD. However, its neuroanatomical assessment in the initial stages is often insufficient due to gradual occurrence of brain atrophy. Functional imaging techniques such as functional MRI (fMRI) and fluorodeoxyglucose positron emission tomography (FDG-PET) usually provide a higher sensitivity to detect brain dysfunctions before the emergence of clinical symptoms [5]. The most observed structural and functional brain changes in early stages of bvFTD target a group of interconnected brain regions, so-called "salience network" (SN), which is associated with socialemotional processing [1]. Taken together, integrating structural and functional brain alterations in bvFTD may increase accuracy of neuroimaging biomarkers and may improve the specificity 
of probable bvFTD criteria. However, the individual neuroimaging studies in bvFTD point to divergent findings due to heterogeneous clinical samples, diversity of imaging modalities, flexible analyses and statistical methods. Thus, a consolidation of the literature is needed to overcome the previous inconsistencies. Quantitative assessment of neural abnormalities using neuroimaging meta-analysis is a favorable tool to overcome such divergence in the bvFTD literature $[6,7]$. There are few previous bvFTD neuroimaging meta-analyses that indicated atrophy and hypometabolism in a wide number of brain regions covering the frontomedial cortex, basal ganglia, anterior insula, and the temporal cortex [8-10]. However, these previous meta-analyses were mostly unimodal i.e., including only VBM experiments, FDG-PET, or resting-state fMRI (rs-fMRI) [8-10], included a low number of patients, various selection criteria, and often used liberal statistical methods (e.g., FDR), which provides a significant error to control for false positive results [11].

Using the best-practice neuroimaging meta-analysis guidelines [6, 7], the present study aimed to revisit the past meta-analytic findings by including multimodal datasets including structural MRI, FDG-PET, and fMRI imaging modalities. In order to elucidate consensus structural and functional regional aberrations in bvFTD, we applied activation likelihood estimation (ALE) meta-analysis based on the largest number of individual bvFTD findings. ALE is the most common applied algorithm among the coordinate-based meta-analysis (CBMA) methods which assess the regional convergence between foci obtained from group comparison experiments [12]. Afterward, we located their brain co-activation patterns using meta-analytic co-activation mapping (MACM) [13] and resting-state functional connectivity (RSFC) [14] to reveal the networks connected to the meta-analytic obtained regions. We performed both analyses since MACM reveals the co-activation pattern of various regions in task-based experiments, while RSFC demonstrates the functional connectivity (FC) of each region in resting-state fMRI. Moreover, we performed hierarchical clustering analysis based on their pairwise RSFC profile and functional decoding of the convergent clusters to reveal the subnetworks between convergent regions and assess the functions associated with these regions, respectively. Finally, we assessed functional characteristics of the identified regions using the BrainMap dataset [15]. 


\section{Methods}

The present large-scale CBMA was performed following the recently developed best-practice guidelines for neuroimaging meta-analyses [6, 7] and adhering to the Preferred Reporting Items for Systematic Reviews and Meta-Analyses (PRISMA) [16]. The protocol for this study was preregistered based on the International Prospective Register of Systematic Reviews (PROSPERO, code: CRD42020127902).

\subsection{Search and Study Selection}

We searched PubMed and Embase databases to retrieve potentially relevant studies in May 2020 using the following keywords: (behavioral variant frontotemporal dementia OR bvFTD OR frontal variant frontotemporal dementia OR frontotemporal dementia OR picks disease) AND (functional magnetic resonance imaging OR fMRI OR voxel-based morphometry OR VBM OR positron emission tomography OR PET). To avoid missing any additional relevant studies, we also traced the references of the included studies and previous reviews/meta-analyses on bvFTD. After removing the duplicates, unique records were independently reviewed by two authors (A.K. \& M.M.), first by screening the abstracts and then, for the possible relevant ones, by reading their full-texts. In case of discordance between two raters, the disagreement was settled by another expert reviewer (T.K.). Studies were included if they met the following eligibility criteria: (1) were reported in a peer-reviewed original article and not as a conference abstract; (2) were included bvFTD patients who were clinically diagnosed using the most reliable guidelines at the time of publication [3, 17] and compared them with healthy controls (HC); (3) both bvFTD and control groups were free of any additional major comorbidities such as amyotrophic lateral sclerosis or other neurological disorders; (4) each group was included with $\geq$ six participants; (5) neuroimaging methods including VBM, fMRI (resting-state or task-based), and FDG-PET were used; (6) the analysis had to be whole-brain and not limited to a region of interest (ROI) or seedbased FC; and (7) the results of between-group contrasts were reported as peak coordinates in a standard space (i.e., Montreal Neurological Institute [MNI] or Talairach), or were provided by authors upon our request, in case they were not mentioned in the individual studies. 


\subsection{Data Extraction}

Three independent authors (T.K. \& A.K. \& M.M.) reviewed the full-texts of the included studies and extracted their relevant data, including bibliographic information, average age and years of education, type of imaging modality (VBM, FDG-PET, rs-fMRI, task-based fMRI (t-fMRI)), and the peak coordinates $(\mathrm{x}, \mathrm{y}, \mathrm{z})$ of each experiment in MNI or Talairach space. Of note, in CBMA, "experiments" refers to the individual group comparison contrasts (e.g., bvFTD > HC, and HC > bvFTD) reported in a "study", which indicates an individual publication [6]. Prior to analyses, we transformed Talairach coordinates into the MNI space for all coordinates to set them in the same reference space[18]. In order to avoid pooling the data from overlapping samples, which would potentially influence the results, we identified publications with partially/fully overlapping samples, and merged their data to minimize the within-group effect, as suggested previously [19].

\subsection{Activation likelihood estimation analysis}

We used the revised version of the ALE method as implemented in MATLAB to test the spatial convergence of the reported coordinates, against the null hypothesis of randomly distributed findings across the brain [12]. In this approach, for each experiment the foci are modeled as 3D Gaussian kernels representing the uncertainty about the location of peak coordinate. The uncertainty is assumed to be greater for experiments with smaller sample sizes, which is characterized by a higher full width at half maximum (FWHM) of Gaussian kernels. Subsequently, a "modeled activation" (MA) map is created for each experiment by combining all of their convolved foci. Next, taking the union of MA maps for all of the included experiments results in an ALE score map, which represents voxel-wise likelihood of convergent findings at each particular brain location. Finally, to distinguish true convergence from random overlap, the ALE score map is statistically tested against a null-distribution of randomly distributed findings $[12,19]$, at $\mathrm{p}<0.05$ cluster level family-wise error (cFWE) corrected for multiple comparisons.

The experiments were further categorized by their effect direction (in-/decreases) and imaging modalities, and then separate ALE meta-analyses were performed on four subsets of the experiments, which included the minimum required number of experiments for sufficient power ( $\geq 15$ ) [11]. Our analyses included (i) all the experiments together, (ii) experiments reporting decreases in activity/connectivity, metabolism or grey matter volume (HC > bvFTD), (iii) VBM 
experiments, and (iv) combination of functional imaging (FDG-PET, rs-fMRI, t-fMRI) experiments. The other sets of experiments were less than 15 and thus, no analysis was conducted based on them.

\subsection{Meta-analytic co-activation mapping}

To characterize task-based functional connectivity patterns of the identified convergent brain regions in the all-effects ALE analysis, we used MACM [13]. In this approach, we used the data curated in the BrainMap database [15] derived from more than 10,000 task-based functional neuroimaging studies on healthy individuals, to meta-analytically identify regions that are coactivated across a range of different tasks with the seed region. More specifically, whole-brain peak coordinates of all studies that reported at least one focus of activation within the respective seeds were analyzed using the ALE algorithm and thresholded at $\mathrm{p}<0.05$, FWE cluster-level corrected (voxel-wise, cluster-forming threshold: $\mathrm{p}<0.001$ ) to detect co-activations areas with the meta-analytic identified regions.

\subsection{Resting-state functional connectivity analysis}

We also investigated the task-free FC patterns of the convergent meta-analytic clusters using RSFC analysis. Here, the data from 192 healthy individuals obtained from the Enhanced Nathan Kline Institute - Rockland Sample was used [20]. BOLD images were acquired using a Siemens TimTrio $3 \mathrm{~T}$ scanner (gradient-echo EPI pulse sequence, $\mathrm{TR}=1.4 \mathrm{~s}, \mathrm{TE}=30 \mathrm{~ms}$, flip angle $=65$, voxel size $=2.0 \mathrm{~mm} \times 2.0 \mathrm{~mm} \times 2.0 \mathrm{~mm}, 64$ slices $)$ while the subjects were in a resting condition and instructed to keep their eyes open. The physiological and movement artifacts were removed from the images using FMRIB's ICA-based Xnoiseifier (version 1.061, as a component of FSL 5.0.9) [21], and additional preprocessing was performed using SPM8 (Wellcome Trust Centre for Neuroimaging, London) and in-house MATLAB scripts, following previously employed processing procedures [22]. To estimate RSFC, Pearson's correlation coefficients of the average time-course of each seed with the time-series of all other gray-matter voxels was calculated and then transformed into Fisher's z scores, which were entered in a second-level ANOVA for group analysis in which age and gender were included as covariates of no-interest. Finally, non-parametric permutation testing was performed to threshold the obtained RSFC maps at $\mathrm{p}<0.05$, FWE corrected for multiple comparisons on the cluster level. 


\subsection{Overlap of task-based and task-free functional connectivity}

The maps of task-based (i.e., MACM) and task-free (i.e., RSFC) connectivity for each seed region were overlapped to estimate their consensus connectivity map and identify the areas that show robust task-independent as well as task-dependent FC. In particular, we performed conjunction analysis across the MACM and RSFC maps [23].

\subsection{Anatomical labeling and visualization}

To identify the macrostructural label and cytoarchitectural characteristics of the respective brain regions identified in the ALE or functional connectivity analyses, we used the SPM Anatomy Toolbox, V3 [24]. We used MRIcroGL v 1.2.2 [25]for brain visualizations.

\subsection{Hierarchical cluster analysis}

We performed hierarchical clustering based on the pairwise RSFC patterns of identified metaanalytic regions to group them into clusters that contain regions that are as homogenous as possible while maximally distinct from other clusters. To calculate the RSFC matrix, partial temporal correlation between the time series data of all regions was estimated in FSLNets toolbox (http://fsl.fmrib.ox.ac.uk/fsl/fslwiki/FSLNets) [26], followed by Fisher's ztransformation of correlation coefficients for each pairwise connection which were submitted to one-sample t-tests. The resulting $\mathrm{t}$ values, reflecting connection strength, as well as consistency across the samples, were z-transformed into units of the standard normal distribution. The obtained RSFC matrix was then fed into the Ward hierarchical clustering. Of note, to preserve the full pattern of connectivity profiles, all features entered the analyses without any thresholding for significance. Hierarchical clustering was performed through an agglomerative approach in which clusters initially formed by individual regions are subsequently merged according to their similarity using standardized Euclidean distances and Ward's incremental sum of squares method [27].

\subsection{Functional decoding analysis}

The behavioral domains (BD) of the experiments included in BrainMap database and their associated activation maps were used to explore the functional role of the identified convergent regions [28]. More specifically, within each convergent brain region, the overall a priori chance 
of finding activations across all BDs, $\mathrm{P}$ (Activation), was compared with the probability of its activation during each particular $\mathrm{BD}, \mathrm{P}($ Activation $\mid \mathrm{BD})$, using binomial tests which were corrected for multiple comparisons using the false discovery rate (FDR), and BDs with a significantly higher probability of activating each respective region than average were reported.

\section{Results}

\subsection{Experiments included in the meta-analysis}

After removing duplicate records, we screened 5,047 abstracts and included 73 studies in our meta-analysis (Figure 1, Supplementary Table 1). Of note, among these included papers, 27 studies were performed based on the FRONTIER open dataset and thus, their data were merged to minimize the within-group effect [6]. In addition, eight other studies had partially overlapping samples, and they were pooled together as well. Finally, 31 independent experiments (out of 73 studies) comprising 1672 bvFTD patients and $3884 \mathrm{HC}$ participants were used for ALE analysis. These experiments include VBM $(\mathrm{N}=20)$, t-fMRI $(\mathrm{N}=1)$, rs-fMRI $(\mathrm{N}=2)$ or FDG-PET $(\mathrm{N}=$ 12). Of note, some experiments used more than one imaging modality; therefore, the collective number of experiments included in each imaging modality exceeds the number of total experiments. Overall, patients and controls had a comparable average age and years of education. Decrease in functional/structural experiments (HC > bvFTD) was observed more commonly ( $\mathrm{N}$ $=28)$ and only a few studies $(\mathrm{N}=3)$ had reported increased functional/structural experiments (bvFTD > HC). The excluded studies during reading full papers and the reasons of exclusion are reported in Supplementary Table 2.

\subsection{Convergent regional abnormalities in bvFTD}

Firstly, we assessed the consistent structural and functional abnormalities by pooling all experiments $(\mathrm{N}=31)$, and identified five convergent clusters in the following regions $(\mathrm{p}<0.05$, cFWE) (Supplementary Table 1, Figure 2A): (i) the right amygdala and hippocampus (max coordinate: $24,-6,-14 ; 240$ voxels), with $17.8 \%$ located in CA1, $13.1 \%$ in centromedial amygdala, $11.1 \%$ in dentate gyrus, $11.4 \%$ in ventromedial amygdala, and $7.7 \%$ in basolateral amygdala. Convergence in this cluster was mostly driven by VBM experiments (87.8\%); (ii) the left caudate and subcallosal cortex (max coordinates: $-4,12,-12 ; 534 \mathrm{v} \%$ ) and area s24 (5.8\% oxels), including regions in area 25 (13.8\%), area 33 (8.3\%), area Fo2 (4.8). Experiments 
using VBM (54.6\%), FDG-PET (28.5\%), or both VBM and FDG-PET (16.8\%) contributed to determine this cluster; (iii) bilateral paracingulate gyrus and anterior cingulate cortex (ACC) (max coordinates: $10,34,28 ; 400$ voxels), with $14.1 \%$ of its volume located in area $24 \mathrm{c}, 11.4 \%$ in area $\mathrm{p} 32,10.4 \%$ in area $\mathrm{p} 24 \mathrm{ab}$, and $2.6 \%$ in area 33. Convergence in this cluster was driven by VBM (53.8\%), FDG-PET (33.6\%), both VBM and rs-fMRI (7.3\%), or both VBM and FDG-PET $(5.1 \%)$ experiments; (iv) bilateral paracingulate gyrus extending to small portions of the medial orbitofrontal cortex (max coordinates: $0,36,-10 ; 163$ voxels), with 52.5\% located in area s32, $2.19 \%$ in area s24, $8.9 \%$ in area p24ab, and 3.6\% in area p32. This cluster almost entirely resulted from the convergence of VBM experiments (98.5\%), with only $1.3 \%$ contribution of FDG-PET experiments; (v) the left anterior insular cortex (AIC) extending to frontal orbital cortex (max coordinates: -32, 22, 4; 173 voxels), with 30.9\% located in area Id6, 29.9\% in area Id7, and $9.6 \%$ in area OP8. In this cluster the highest contribution was from VBM experiments (72.3\%), but FDG-PET experiments also contributed to identify this cluster (27.2\%). As most of the included experiments had reported decrease contrasts (HC > bvFTD), performing ALE analysis on these experiments only showed very similar convergent clusters $(\mathrm{p}<0.05$, cFWE) (Supplementary Table 1, Figure 2B).

Next, we performed separate ALE analyses based on imaging modality using VBM $(\mathrm{N}=$ 21) or combination of functional imaging $(\mathrm{N}=17)$ experiments. The ALE analysis on VBM experiments revealed clusters of convergence in the amygdala and hippocampus (max coordinates: $24,-6,-14 ; 304$ voxels), paracingulate gyrus and frontal medial cortex (max coordinates: $0,36,-10 ; 203$ voxels), as well as the AIC and frontal orbital cortex (max coordinates: $-32,22,4 ; 100$ voxels) ( $<<0.05$, cFWE) (Supplementary Table 1, Figure 2C). The location of these clusters corresponded to the first, third and fifth clusters of the all-experiments analysis, respectively, but was smaller in size. Confining the analysis to the functional experiments (i.e., FDG-PET, rs-fMRI, and t-fMRI) demonstrated three significant clusters in the left caudate and accumbens (max coordinates: -8 10 0; 276 voxels), paracingulate gyrus and ACC (max coordinates: 1034 26; 296 voxels), and another more rostral region in the ACC (max coordinates: 414 34; 156 voxels). The last cluster, unlike the other two did not correspond to any of the regions identified in the all-experiments analysis. Convergence in the significant clusters of functional analysis was mainly driven by FDG-PET $(67.1 \%-100 \%$ contribution) and rs-fMRI 
experiments (11.1\%-32.9\%), while t-fMRI experiments had no contribution ( $\mathrm{p}<0.05$, cFWE) (Supplementary Table 1, Figure 2C).

\subsection{Connectivity patterns of the identified convergent regions}

MACM and RSFC analyses pointed to the joint networks that are connected to the obtained consistent regions (Figure 3, Supplementary Figures 1 and 2). The overlap of MACM and RSFC maps revealed significant task-based and task-free co-activation of the amygdala and hippocampus with the striatum, thalamus, fusiform gyrus, inferior frontal gyrus, and midline frontal regions. MACM analysis demonstrated that this region has significant co-activation with the AIC. In addition, RSFC analysis showed additional task-free connectivity of this region with the entorhinal cortex, superior and middle temporal gyri, precuneus, posterior cingulate cortex, and cerebellum.

The convergent cluster in the left caudate and subcallosal cortex was associated with the striatum, thalamus, AIC, midline frontal regions, posterior cingulate gyrus, and cerebellum in both MACM and RSFC analyses, but with the superior lateral occipital cortex, and middle temporal gyrus only in task-free analysis, and the left superior parietal lobule and parietal operculum only in task-based analysis. The rostral paracingulate and frontal medial cortex cluster showed task-based coactivation and RSFC with the medial frontal regions, posterior cingulate cortex, precuneus, hippocampus, amygdala, and superior lateral occipital cortex, but was also functionally connected to the medial thalamus, AIC, Heschl's gyrus, and cerebellum only in RSFC analysis. Most of the significant regions for this cluster in the MACM analysis were also observed in the RSFC analysis.

The more caudal significant cluster in the paracingulate and ACC similarly functionally connected to the medial frontal regions, AIC, striatum, thalamus, middle and inferior frontal gyri, and posterior cingulate cortex in MACM and RSFC analyses, but additionally revealed RSFC with the cerebellum, and meta-analytic co-activation with the superior parietal lobule and right angular gyrus. The AIC cluster was functionally connected with the paracingulate gyrus and ACC, striatum, thalamus, middle and inferior frontal gyri, superior parietal lobule, and different regions within the cerebellum during both task and rest. In addition, the AIC showed only taskbased functional connectivity with the precentral gyrus, fusiform gyrus, and superior parietal 
lobule, and RSFC with the temporal pole, Heschl's gyrus, intracalcarine cortex, and lingual gyrus. All these results were corrected for cFWE.

\subsection{Hierarchical clustering of convergent findings}

To identify functionally coherent sub-networks of the identified consistent regions, we performed hierarchical clustering analysis based on their pairwise RSFC profile. In this analysis, at the first level, we identified two main sub-networks, namely the insula-amygdala network, and the cingulo-striatal network including the ACC, paracingulate cortex, frontal medial cortex, subcallosal cortex, and striatum. The latter was grouped into two additional sub-networks at the second level: one including the two clusters located in the medial frontal lobe, and the other one including the convergent cluster in the striatum and subcallosal cortex (Figure 4).

\subsection{Functional decoding of convergent clusters}

Our forward-inference functional decoding of the identified regions using the BrainMap database demonstrated their significant involvement in emotional processing, interoception, reward processing, higher-order cognitive functions, as well as olfactory and gustatory perception (Figure 5). More specifically, the amygdala/hippocampus cluster was activated in olfactory perception, processing of negative and positive emotions, and reward processing. The convergent cluster in the left caudate and subcallosal cortex was mostly associated with reward processing, gustatory perception, cognitive reasoning, and sexual interoception. Gustatory perception and reward processing were also associated with the activations in the rostral paracingulate gyrus / frontal medial cortex cluster. The more caudal paracingulate gyrus and the ACC cluster was more likely to be activated in cognitive reasoning and response to rewards. Finally, the AIC was associated with thermal interoception, processing of disgust, and language semantics. All these results were corrected for FDR.

\section{Discussion}

We performed a large-scale CBMA on both structural and functional brain studies on bvFTD, nearly a decade after the revision of its diagnostic criteria, and found consistent abnormalities in the five clusters including the AIC, ACC, paracingulate cortex, subcallosal cortex, striatum, amygdala, and anterior hippocampus in patients with bvFTD compared to healthy subjects. 
These regions predominantly showed decreased grey matter volume, functional hypoactivation, or dysconnectivity, to various degrees in each region. While the abnormalities in the striatum were mainly functional, structural abnormalities were more predominant in the AIC, amygdala, and anterior hippocampus, and midline frontal regions were both functionally and structurally impaired. In addition, we used MACM and RSFC analyses to characterize the connectivity pattern of the convergent regions and observed significant co-activation of them with each other, and with additional brain regions including the thalamus, lateral prefrontal cortex, and association cortices in the parietal lobe. Next, using hierarchical clustering of the convergent regions, we classified them into two main groups: one including the AIC, amygdala, and hippocampus, and the other one including the midline frontal areas and striatum. Moreover, functional decoding analysis showed involvement of the former first set of regions in emotional processing, and of the latter in reward processing and higher-order cognitive functions.

\subsection{The key role of the salience network in bvFTD}

The cortical layer 5b of AIC and pregenual ACC (pgACC) contains a specialized type of large spindle-shaped projection neurons called von Economo neurons (VEN), which are presumed to be involved in social cognition and self-awareness [29]. The number of these neurons in the AIC and pgACC is significantly depleted in patients with bvFTD [30-32], parallel to clinical severity of bvFTD, and even in the absence of gross atrophy of these regions [29]. Accordingly, histopathological and imaging studies have suggested that the AIC and pgACC are among the earliest atrophied regions in bvFTD [33]. These regions are the key hubs of the SN, which guides behavior in response to the perceived salience of current external/internal events, i.e., their significance for the survival of the individuals [32]. In this network, the AIC and pgACC play distinct, but interdependent roles, acting as afferent ("sensory") and efferent ("motor") hubs of the SN, respectively. More specifically, the AIC detects and represents subjective emotional, homeostatic, social, and motivational salience of the immediate environmental or bodily states [34, 35], and the ACC initiates goal-directed behaviors in response to these salient stimuli [36]. Several studies have reported decreased FC within the SN in patients with bvFTD [37]. It is well-documented that the main hubs of the SN can lead to the main characteristic symptoms of bvFTD, including impaired emotional processing and social cognition, disinhibition, executive dysfunction, and apathy. 
Impaired emotional recognition is a common symptom in bvFTD and has been reported for different types of emotional stimuli, including facial expressions, non-verbal emotional sounds, or music [38]. This impairment is selective for negative emotions, and patients' ability for recognizing and reacting to positive emotions is often spared [39] or even disproportionately increased [40]. In addition to emotional recognition, patients with bvFTD have deficits in suppressing emotions, generating emotions, and perceiving self-conscious emotions [41]. Decreased grey matter volumes of the amygdala and AIC have been reported in bvFTD patients with impaired emotional recognition of facial expressions [42]. Amygdala atrophy, similar to AIC, occurs early in the course of bvFTD [43], and was consistently reported in our included studies. The amygdala has reciprocal connections to the ventral striatum, as well as limbic and paralimbic brain regions [43], and has a central role in recognition of emotions, but also in reward processing, motivation, attention, learning and memory [44]. In addition, the amygdala along with the temporal pole, ventral striatum, and thalamus, is responsible for the SN functions that include providing the AIC with the information about socio-emotional valence of the external and internal world. The information from these different sources is integrated in the AIC, where the salience of the current state is determined/represented, and passed on to more downstream regions [36].

Lack of empathy, i.e., impaired affective social cognition, is a core diagnostic feature of bvFTD [3] and is closely related to the deficits in emotional processing [45]. Empathy indicates an ability of identifying and sharing emotions and needs of other individuals [46]. Deficits in empathy can have a detrimental effect on the relationships of patients with their relatives and caregivers [47]. In addition, lack of empathy, and impaired social cognition in general, can result in disinhibition of socially inappropriate behaviors. More specifically, disinhibition might be a consequence of patients' inability to correctly identify social and emotional signals and their associated punishments/rewards, thereby neglecting the negative consequences of their own social acts [45]. Recent neuroimaging meta-analyses on healthy individuals have shown that empathy is consistently associated with activation of regions including the AIC, amygdala, ACC, thalamus, and lateral frontal regions [48, 49]. In addition, individual differences in socioemotional sensitivity has been shown to correlate with FC of the SN [50], and interestingly, socio-affective training aimed at improving empathy and compassion is associated with plasticity of the AIC [51]. Several neuroimaging studies on the neural correlates of empathy and social 
cognition in bvFTD patients have pointed out to abnormalities in the similar regions, such as the insula, thalamus, amygdala, inferior frontal gyrus, lateral orbitofrontal cortex, and medial frontal regions including subcallosal and midcingulate cortex [40, 50, 52, 53]. Interestingly, similar regions are involved in impaired socio-emotional dysfunctions of other neuropsychiatric disorders such as autism spectrum disorder [54], conduct disorder [55], and schizophrenia [56].

Based on the 'simulation theory of empathy', humans use their own mind as a model to predict and understand the thoughts and feelings of others [34]. Therefore, and as self-awareness of affective states largely relies on interoception, i.e., awareness of internal bodily homeostatic state, it has been suggested that interoception has an important role in empathy [34, 57]. These two closely related functions are presumed to be mediated by the AIC, where dual and corresponding mappings for subjective and empathetic feeling states are represented [46]. In line with this hypothesis, a fMRI study demonstrated that when subjects are interoceptively aware (attending to their heartbeats), they show a higher empathy-related brain activity in the AIC after observing emotionally valent facial expressions [57]. Patients with bvFTD display impaired interoception, as has been shown by their decreased performance in the heartbeat detection task [58], or their lower sensitivity to pain and temperature [59], which interestingly is associated with the atrophy of insula and fronto-temporal regions [58]. The lack of empathy in bvFTD patients could therefore be partially attributable to the impairments in interoception, which are due to AIC dysfunction.

Based on our findings in the AIC, it is worthy to note that this convergent cluster was located more on the dorsal surface of the left AIC. This region, as suggested by previous studies and our functional decoding, is more involved in cognitive control and semantic functions [60], as opposed to the right and ventral AIC, which has a key role in socio-emotional processing [61]. Although we only include studies on bvFTD patients, this finding suggests that some studies might include bvFTD patients with concomitant language abnormalities or semantic variants of FTD. Nevertheless, the segregation of functions in the dorsal and ventral AIC is not as clear-cut and different functions of AIC have shown to converge on its dorsal surface [62]. In addition, our functional decoding analysis showed that the left dorsal AIC is more likely to be activated in interoception than in language functions, and as mentioned above, interoception is a key component of empathy. Of note, previous neuroimaging meta-analyses of empathy have also 
shown activation of both left and right, as well as ventral and dorsal AIC in response to emotional stimuli [49].

\subsection{Limbic system abnormalities in bvFTD result in executive dysfunction and apathy}

The ACC, as the efferent hub of SN, coordinates initiation of appropriate behaviors in response to the states that are emotionally, socially, or homeostatically significant [36]. This function is mainly accomplished by switching the brain activity from the default mode network (DMN) to the central executive network (CEN), [63], which refers to the brain areas that are engaged during executive functions, i.e., cognitively demanding tasks that require sustained attention, including working memory, problem solving, planning, inhibiting, and development or implementation of strategies [64]. Executive dysfunction is a prominent symptom and a key diagnostic feature of bvFTD, which affects many domains of higher order brain functions, and contributes to development of apathy or inertia (see below) [65]. Meta-analytic studies have suggested that in healthy individuals, the frontoparietal and subcortical structures such as the dorsolateral prefrontal cortex, superior parietal lobules, dorsal ACC, thalamus, and striatum are involved in executive functions [64]. Accordingly, executive dysfunction in bvFTD patients is associated with atrophy of anterior cingulate and midcingulate gyri, medial frontal cortex, and lateral prefrontal cortex [66]. In the present meta-analysis, we found consistent abnormalities in the ACC and caudate nucleus in bvFTD patients. These regions were grouped together in the hierarchical clustering, and showed FC with each other, and other key regions of the CEN including the prefrontal cortex and superior parietal lobule. However, we found no consistent abnormality in the lateral frontal and parietal regions classically associated with executive functions. This finding suggests that in addition to the primary deficits in the ACC and striatum, executive dysfunction in bvFTD patients might be secondary to their inability to engage these lateral cortical regions due to dysfunction in the ACC and SN. Furthermore, as impairments in classical executive functioning tasks occur later in the course of bvFTD [67], some patients may not have yet developed abnormalities in lateral regions of the CEN, making it less likely to be identified consistently across the literature.

Psychopathology of apathy or inertia involves impairments in motivation, initiation and planning/execution [2]. Although all three components are dysfunctional in bvFTD patients, lack of motivation is the most prominent abnormality, which contributes to apathy [68]. Motivation 
refers to the ability of associating positive or negative affective signals with the value of actions, and attempting to maximize value by seeking rewards and avoiding punishments [69]. In this context, abnormal reward processing can lead to a lack of motivation, both by reducing the inclination to perform and complete tasks and ability to comprehend the consequences of future actions [70]. Recent meta-analysis has shown that in healthy individuals, the striatum, insula, amygdala, thalamus, parahippocampal gyrus, and medial frontal regions such as the ACC are involved in reward processing [71]. We found convergent abnormalities in many of these reward-responsive regions including the left caudate, amygdala, paracingulate cortex, frontal medial cortex, and ACC. Interestingly, motivational deficits in bvFTD patients are associated with atrophy of the orbitofrontal cortex and ACC [72]. These findings suggest that lack of motivation in bvFTD patients occurs as a result of their decreased sensitivity to rewards and punishments, due to dysfunction of the ACC, orbitofrontal cortex, and striatum [70].

\subsection{The role of medial temporal lobe atrophy in amnestic features of bvFTD}

Classically, bvFTD has been mainly described as a predominantly behavioral disorder with less episodic memory impairment [3]. However, patients with bvFTD, like other dementia syndromes may have deficits in encoding and retrieval of autobiographical memories, comparable to that of $\mathrm{AD}$ [73]. It has been reported that impaired episodic memory functions in bvFTD patients is attributable to their executive dysfunction, i.e., their inability to properly monitor topics and events, check the relevance of incoming memories, and inhibit competing memories, due to frontal lobe abnormalities [33]. Although executive dysfunction can be a contributing factor to amnesia, it has been suggested that similar to AD, amnestic bvFTD patients have atrophy or dysfunction of the hippocampus and other medial temporal lobe (MTL) structures [73, 74]. Similarly, we found convergent atrophy in the CA1 and dentate gyrus of the right anterior hippocampus. This finding is rather new than previous neuroimaging meta-analyses on bvFTD, which reported no convergent abnormalities in the MTL $[4,8,10]$. These findings, however, suggest that MTL atrophy and impaired episodic memory exist in bvFTD, and therefore intact memory function may not constitute a suitable differentiating factor between bvFTD and AD [75]. An alternative explanation might be that as both bvFTD and AD are often diagnosed using clinical diagnostic criteria that are not certain [76, 77], a portion of clinically-diagnosed bvFTD patients, when assessed histopathologically, may actually have AD pathology (e.g., 12 out of 63 
patients [76]), or that frontotemporal lobar degeneration (FTLD) and AD cover a spectrum of neurodegenerative disorders with some bvFTD patients also having underlying AD pathology (and vice versa) [78].

\subsection{Potential strengths and limitations}

We replicated some of the findings of previous meta-analyses on bvFTD in the frontomedial areas, AIC, and striatum, but in addition, we identified a cluster of convergence in the amygdala and hippocampus, probably by virtue of a higher number of structural and functional experiments and increased meta-analytic power, On the other hand, we found no convergence in some of the regions commonly reported in previous meta-analyses, namely lateral frontal cortical areas and thalamus. Of note, we used a stringent method of multiple comparison correction (i.e., cFWE), In addition, including both structural and functional experiments from various imaging modalities allowed us to identify regions that are both structurally and functionally impaired.

Our main limitation, which was in fact inherited from the included studies, was the heterogeneity of clinical samples, particularly with regard to the severity of symptoms, histopathological subtypes, and molecular etiology of bvFTD. Several studies have suggested that distinct pathological or genetic subtypes of frontotemporal lobar degeneration (FTLD) have different neuroanatomical correlates [79]. These subtypes are often difficult to determine, and therefore, very few studies had investigated them separately, preventing us from doing subgroup analyses on those specific subtypes. In addition, as most of the included studies had diagnosed bvFTD using the clinical criteria, it is possible that some of the patients had other disorders characterized by symptoms that can overlap with bvFTD, such as AD or other neuropsychiatric disorders [80]. Finally, a methodological limitation of our meta-analysis, which is common to all CBMA methods, was that the pooling of findings was based on the peak coordinates of significant regions. This method essentially ignores the volume and extent of clusters. Alternatively, image-based meta-analytic methods that are based on unthresholded statistical maps can overcome this issue, when enough number of experiments are available [81]. We also advocate for original neuroimaging studies to share their results as unthresholded statistical maps on open online platforms. 


\section{Conclusion}

Using a large-scale ALE meta-analysis on both structural and functional brain studies of bvFTD and following the best-practice neuroimaging meta-analysis guidelines, we found consistent abnormalities in the AIC, paracingulate cortex and ACC, amygdala, hippocampus, and basal ganglia, across the literature. The MACM and RSFC maps revealed a significant overlap of taskbased and task-free co-activation of the convergent clusters identified based on ALE metaanalysis with regions such as the striatum, thalamus, midline frontal regions, AIC, entorhinal cortex, posterior cingulate cortex, and cerebellum. Moreover, hierarchical analysis of the convergent clusters identified two functionally coherent sub-networks of the identified consistent regions, namely the insula-amygdala network and the cingulo-striatal network. Functional decoding of these regions using forward-inference demonstrated a significant contribution of these clusters processing of negative and positive emotions, interoception, reward processing, higher-order cognitive functions, as well as olfactory and gustatory perception. Overall, these findings suggest a central role of the $\mathrm{SN}$ and regions connected to it in the pathology of bvFTD, in line with previous models of bvFTD pathophysiology [36]. The AIC, as the afferent hub of the $\mathrm{SN}$, is involved in detecting the emotional, homeostatic, social, and motivational salience of internal/external environment via inputs from regions such as amygdala. Thus, dysfunctions of the AIC and amygdala in bvFTD patients, can impair their socio-emotional processing, and may lead to disinhibition of socially inappropriate behaviors, and a lack of empathy. The midline frontal regions, and in particular pgACC, act as the efferent hub of the $\mathrm{SN}$ and in response to salient stimuli direct the brain resources towards a state where it can proportionally respond to these stimuli. In addition, these regions, along with the basal ganglia and amygdala, have a key role in reward processing. Hence, the abnormalities of midline frontal regions, basal ganglia, and amygdala in patients with bvFTD, may be responsible for their executive dysfunction, as well as apathy, primarily through a lack of motivation. In addition, we also observed convergent atrophy

of anterior hippocampus in bvFTD patients, challenging the classical view that bvFTD is a predominantly behavioral disorder, sparing episodic memory, and suggesting that perhaps hippocampal atrophy and amnestic symptoms may not suitably differentiate bvFTD and AD. In general, our results highlighted a crucial role of the salience network and subcortical regions in pathophysiology of bvFTD. 


\section{Acknowledgements}

National Institute of Mental Health (R01-MH074457), the Helmholtz Portfolio Theme "Supercomputing and Modeling for the Human Brain," and the European Union's Horizon 2020 Research and Innovation Programme under Grant Agreement No. 945539 (HBP SGA3); NIH grant K99-AG065457 to LP. 


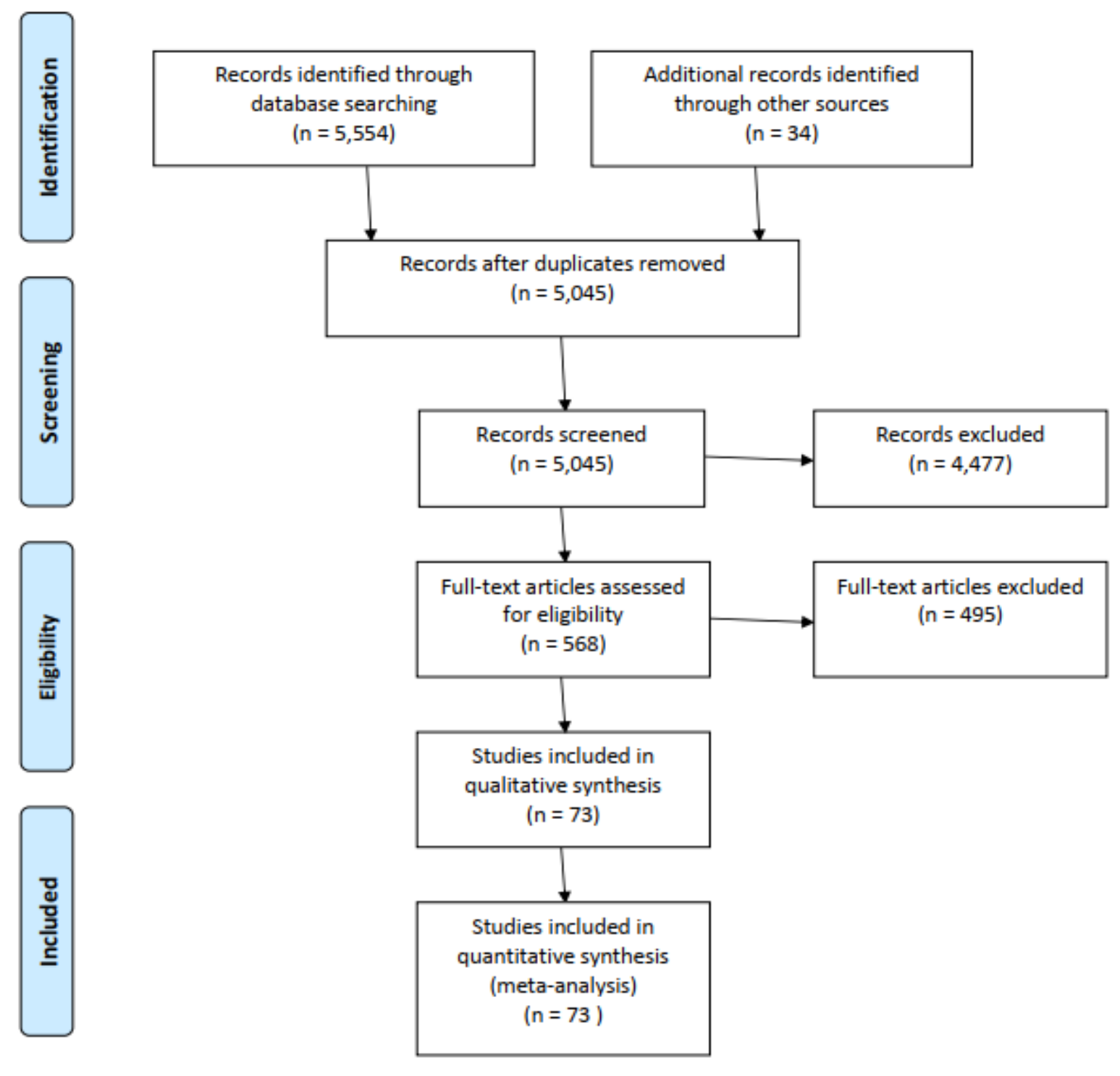

Figure 1. PRISMA flowchart of study selection. PRISMA: Preferred Reporting Items for Systematic Reviews and Meta-Analyses. 
A. ALE analysis based on differences between bvFTD and Controls
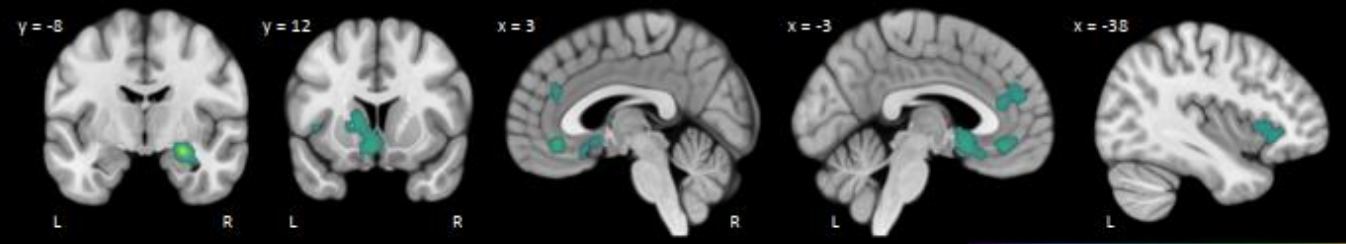

B. ALE analysis based on group contrast (bvFTD < Controls)
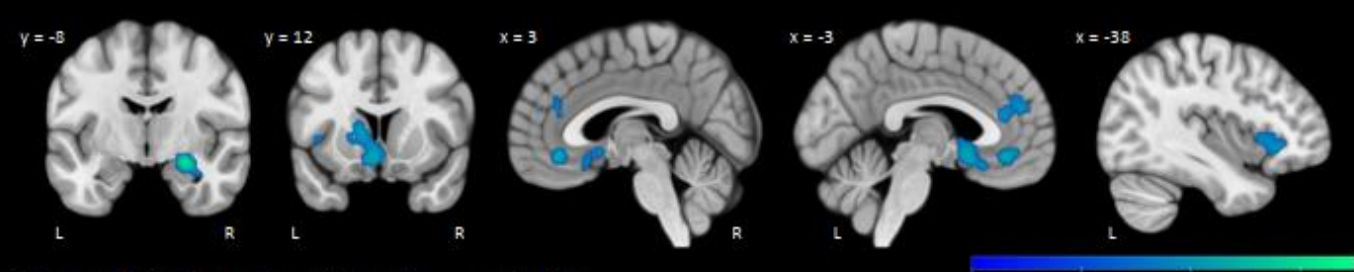

C. ALE analysis based on imaging modality
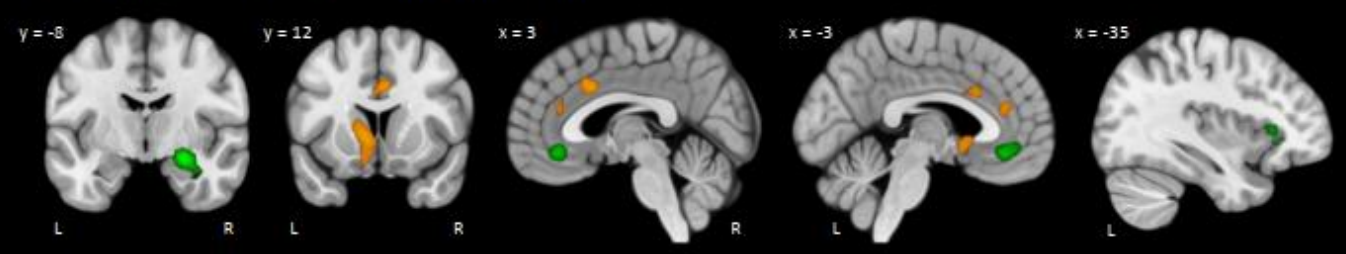

Structural Experiment:

Functional Experiments

Figure 2. Convergence of brain imaging findings in bvFTD compared to healthy controls across all experiments (A), experiments reporting atrophy/hypoactivation (B), and experiments using functional (C, orange) or structural $(\mathrm{C}$, green) modalities. The coordinates are in MNI space. Color bars represent $\mathrm{Z}$ values. ALE: activation likelihood estimation, bvFTD: behavioral-variant frontotemporal dementia, MNI: Montreal Neurological Institute. 


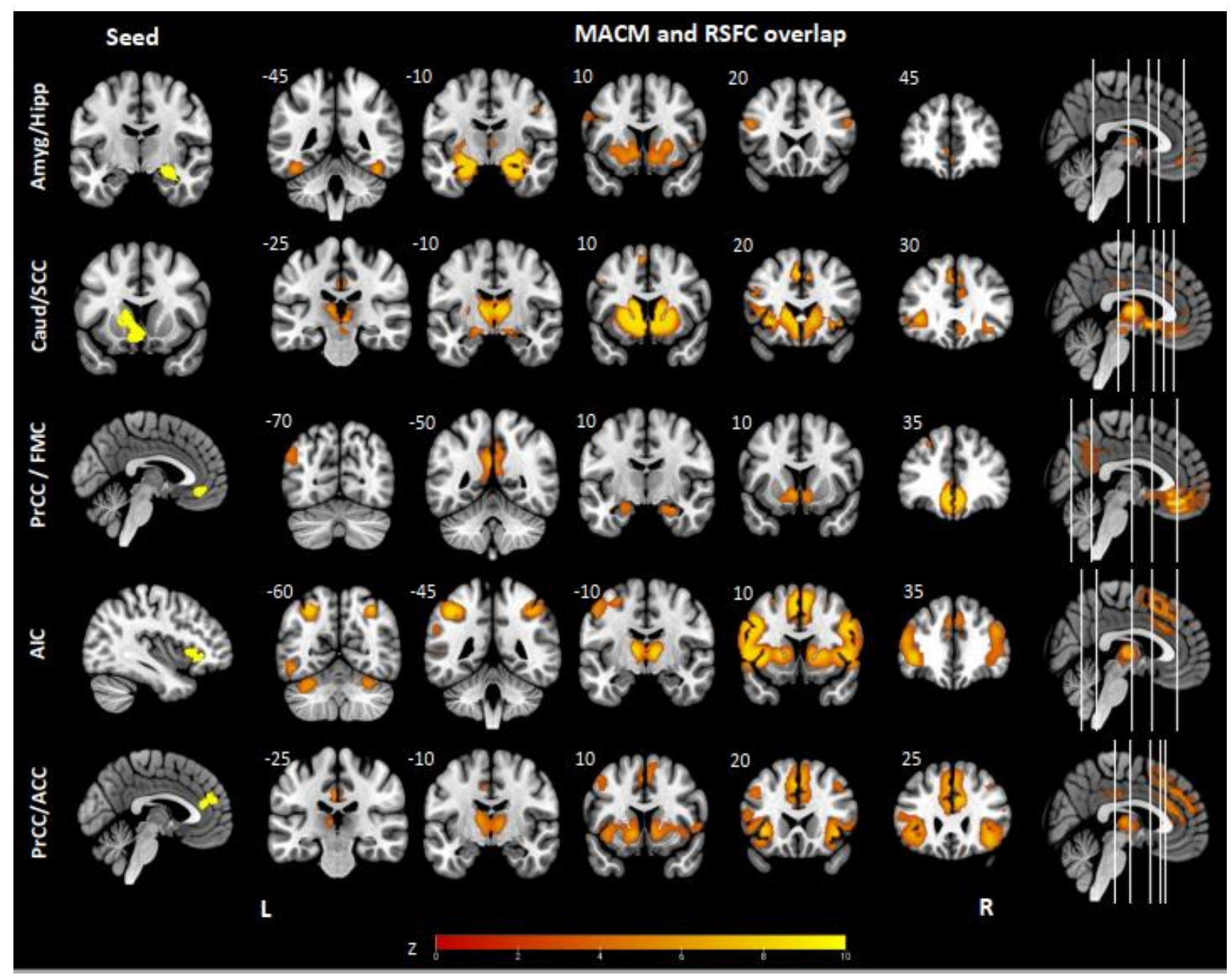

Figure 3. The overlap of resting-state functional connectivity and meta-analytic connectivity maps of convergent regions in the all-effects ALE. The coordinates are in MNI space. MACM: meta-analytic connectivity map, RSFC: resting-state functional connectivity, Amyg: amygdala, Hipp: hippocampus, Caud: caudate nucleus, SCC: subcallosal cortex, PrCC: paracingulate cortex, FMC: frontomedial cortex, AIC: anterior insular cortex, ACC: anterior cingulate cortex, ALE: activation likelihood estimation, MNI: Montreal Neurological Institute. 

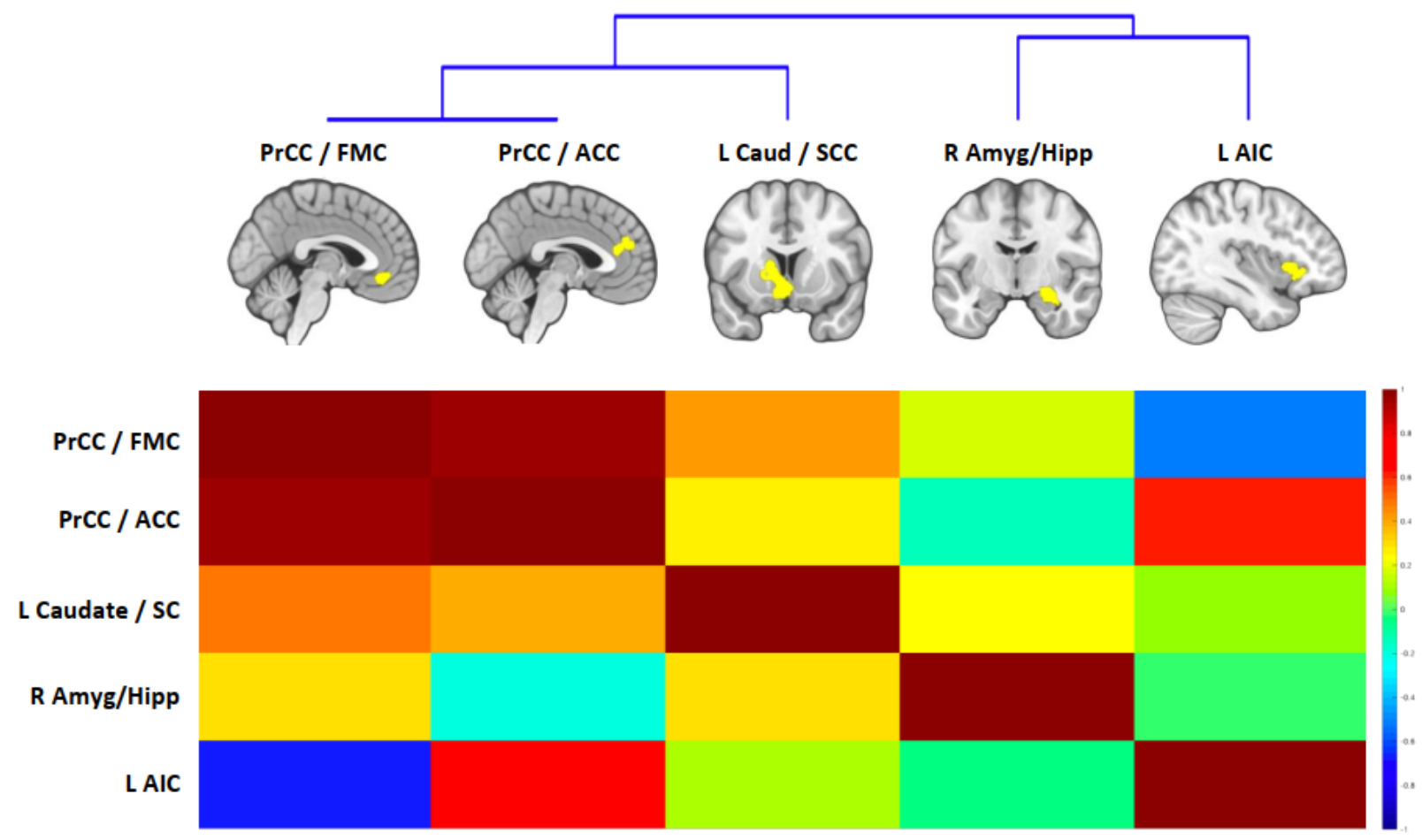

Figure 4. Hierarchical clustering of convergent regions in the all-effects ALE. Below the pair-wise functional connectivity matrix of the convergent regions is shown after Fischer's z-transformation and normalization to the maximum. Amyg: amygdala, Hipp: hippocampus, Caud: caudate nucleus, SCC: subcallosal cortex, PrCC: paracingulate cortex, FMC: frontomedial cortex, AIC: anterior insular cortex, ACC: anterior cingulate cortex, ALE: activation likelihood estimation. 


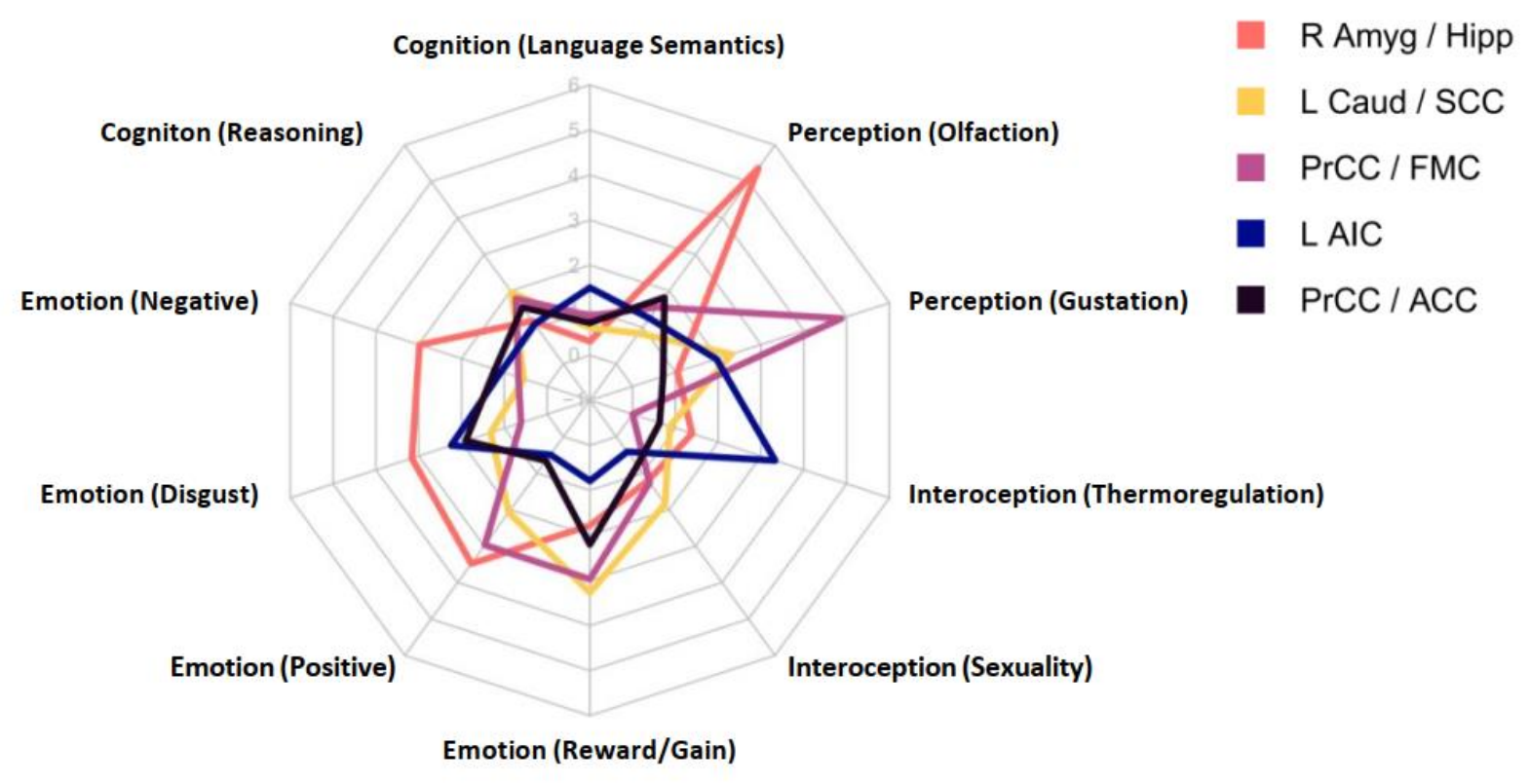

Figure 5. Functional decoding analysis of convergent regions in the all-effects ALE based on BrainMap behavioral domain categories and subcategories. The spider plot values are likelihood ratios. Amyg: amygdala, Hipp: hippocampus, Caud: caudate nucleus, SCC: subcallosal cortex, PrCC: paracingulate cortex, FMC: frontomedial cortex, AIC: anterior insular cortex, ACC: anterior cingulate cortex, ALE: activation likelihood estimation. 


\section{References}

1. Seeley, W.W., Behavioral variant frontotemporal dementia. CONTINUUM: Lifelong Learning in Neurology, 2019. 25(1): p. 76-100.

2. Ducharme, S., et al., Recommendations to distinguish behavioural variant frontotemporal dementia from psychiatric disorders. Brain, 2020. 143(6): p. 1632-1650.

3. Rascovsky, K., et al., Sensitivity of revised diagnostic criteria for the behavioural variant of frontotemporal dementia. Brain, 2011. 134(9): p. 2456-2477.

4. Luo, C., et al., Comparison of gray matter atrophy in behavioral variant frontal temporal dementia and amyotrophic lateral sclerosis: a coordinate-based meta-analysis. Frontiers in aging neuroscience, 2020. 12: p. 14.

5. Gordon, E., J.D. Rohrer, and N.C. Fox, Advances in neuroimaging in frontotemporal dementia. Journal of Neurochemistry, 2016. 138: p. 193-210.

6. Müller, V.I., et al., Ten simple rules for neuroimaging meta-analysis. Neuroscience \& Biobehavioral Reviews, 2018. 84: p. 151-161.

7. Tahmasian, M., et al., Practical recommendations to conduct a neuroimaging meta-analysis for neuropsychiatric disorders. Human brain mapping, 2019. 40(17): p. 5142-5154.

8. Pan, P.L., et al., Gray matter atrophy in behavioral variant frontotemporal dementia: a metaanalysis of voxel-based morphometry studies. Dementia and geriatric cognitive disorders, 2012. 33(2-3): p. 141-148.

9. Pievani, M., et al., Coordinate-based meta-analysis of the default mode and salience network for target identification in non-invasive brain stimulation of Alzheimer's disease and behavioral variant frontotemporal dementia networks. Journal of Alzheimer's Disease, 2017. 57(3): p. 825843.

10. Schroeter, M.L., et al., Conceptualizing neuropsychiatric diseases with multimodal data-driven meta-analyses-The case of behavioral variant frontotemporal dementia. Cortex, 2014. 57: p. 2237.

11. Eickhoff, S.B., et al., Behavior, sensitivity, and power of activation likelihood estimation characterized by massive empirical simulation. Neuroimage, 2016. 137: p. 70-85.

12. Eickhoff, S.B., et al., Activation likelihood estimation meta-analysis revisited. Neuroimage, 2012. 59(3): p. 2349-2361.

13. Laird, A.R., et al., ALE meta-analysis workflows via the brainmap database: progress towards a probabilistic functional brain atlas. Frontiers in neuroinformatics, 2009. 3: p. 23.

14. Shen, K., et al., Network structure shapes spontaneous functional connectivity dynamics. Journal of Neuroscience, 2015. 35(14): p. 5579-5588.

15. Laird, A.R., J.J. Lancaster, and P.T. Fox, Brainmap. Neuroinformatics, 2005. 3(1): p. 65-77.

16. Moher, D., et al., Preferred reporting items for systematic reviews and meta-analyses: the PRISMA statement. PLoS medicine, 2009. 6(7): p. e1000097.

17. McKhann, G.M., et al., Clinical and pathological diagnosis of frontotemporal dementia: report of the Work Group on Frontotemporal Dementia and Pick's Disease. Archives of neurology, 2001. 58(11): p. 1803-1809.

18. Lancaster, J.L., et al., Bias between MNI and Talairach coordinates analyzed using the ICBM152 brain template. Human brain mapping, 2007. 28(11): p. 1194-1205.

19. Turkeltaub, P.E., et al., Minimizing within-experiment and within-group effects in activation likelihood estimation meta-analyses. Human brain mapping, 2012. 33(1): p. 1-13.

20. Nooner, K.B., et al., The NKI-Rockland sample: a model for accelerating the pace of discovery science in psychiatry. Frontiers in neuroscience, 2012. 6: p. 152.

21. Griffanti, L., et al., ICA-based artefact removal and accelerated fMRI acquisition for improved resting state network imaging. Neuroimage, 2014. 95: p. 232-247.

22. Camilleri, J., et al., Definition and characterization of an extended multiple-demand network. NeuroImage, 2018. 165: p. 138-147. 
23. Nichols, T., et al., Valid conjunction inference with the minimum statistic. Neuroimage, 2005. 25(3): p. 653-660.

24. Eickhoff, S.B., et al., A new SPM toolbox for combining probabilistic cytoarchitectonic maps and functional imaging data. Neuroimage, 2005. 25(4): p. 1325-1335.

25. Rorden, C. and M. Brett, Stereotaxic display of brain lesions. Behavioural neurology, 2000. 12(4): p. 191-200.

26. Marrelec, G., et al., Partial correlation for functional brain interactivity investigation in functional MRI. Neuroimage, 2006. 32(1): p. 228-237.

27. Eickhoff, S.B., et al., Co-activation patterns distinguish cortical modules, their connectivity and functional differentiation. Neuroimage, 2011. 57(3): p. 938-949.

28. Müller, V.I., et al., Dysregulated left inferior parietal activity in schizophrenia and depression: functional connectivity and characterization. Frontiers in human neuroscience, 2013. 7: p. 268.

29. Seeley, W.W., J. Zhou, and E.-J. Kim, Frontotemporal dementia: what can the behavioral variant teach us about human brain organization? The Neuroscientist, 2012. 18(4): p. 373-385.

30. Nana, A.L., et al., Neurons selectively targeted in frontotemporal dementia reveal early stage TDP-43 pathobiology. Acta neuropathologica, 2019. 137(1): p. 27-46.

31. Santillo, A.F., C. Nilsson, and E. Englund, von E conomo neurones are selectively targeted in frontotemporal dementia. Neuropathology and applied neurobiology, 2013. 39(5): p. 572-579.

32. Seeley, W.W., et al., Dissociable intrinsic connectivity networks for salience processing and executive control. Journal of Neuroscience, 2007. 27(9): p. 2349-2356.

33. Irish, M., O. Piguet, and J.R. Hodges, Self-projection and the default network in frontotemporal dementia. Nature Reviews Neurology, 2012. 8(3): p. 152-161.

34. Critchley, H.D. and N.A. Harrison, Visceral influences on brain and behavior. Neuron, 2013. 77(4): p. 624-638.

35. Pasquini, L., et al., State and trait characteristics of anterior insula time-varying functional connectivity. Neuroimage, 2020. 208: p. 116425.

36. Zhou, J. and W.W. Seeley, Network dysfunction in Alzheimer's disease and frontotemporal dementia: implications for psychiatry. Biological psychiatry, 2014. 75(7): p. 565-573.

37. Toller, G., et al., Individual differences in socioemotional sensitivity are an index of salience network function. Cortex, 2018. 103: p. 211-223.

38. Omar, R., et al., The structural neuroanatomy of music emotion recognition: evidence from frontotemporal lobar degeneration. Neuroimage, 2011. 56(3): p. 1814-1821.

39. Lough, S., et al., Social reasoning, emotion and empathy in frontotemporal dementia. Neuropsychologia, 2006. 44(6): p. 950-958.

40. Hua, A.Y., et al., Enhanced positive emotional reactivity undermines empathy in behavioral variant frontotemporal dementia. Frontiers in neurology, 2018. 9: p. 402.

41. Van den Stock, J. and F. Kumfor, Behavioural variant frontotemporal dementia: at the interface of interoception, emotion and social cognition. Cortex, 2017. 115: p. 335-340.

42. Kumfor, F., et al., Discrete neural correlates for the recognition of negative emotions: insights from frontotemporal dementia. PloS one, 2013. 8(6): p. e67457.

43. Bocchetta, M., et al., Amygdala subnuclei are differentially affected in the different genetic and pathological forms of frontotemporal dementia. Alzheimer's \& Dementia: Diagnosis, Assessment \& Disease Monitoring, 2019. 11: p. 136-141.

44. Murray, E.A., The amygdala, reward and emotion. Trends in cognitive sciences, 2007. 11(11): p. 489-497.

45. Cerami, C. and S.F. Cappa, The behavioral variant of frontotemporal dementia: linking neuropathology to social cognition. Neurological Sciences, 2013. 34(8): p. 1267-1274.

46. Singer, T., H.D. Critchley, and K. Preuschoff, A common role of insula in feelings, empathy and uncertainty. Trends in cognitive sciences, 2009. 13(8): p. 334-340.

47. Hsieh, S., et al., When one loses empathy: its effect on carers of patients with dementia. Journal of geriatric psychiatry and neurology, 2013. 26(3): p. 174-184. 
48. Bzdok, D., et al., Parsing the neural correlates of moral cognition: ALE meta-analysis on morality, theory of mind, and empathy. Brain Structure and Function, 2012. 217(4): p. 783-796.

49. Fallon, N., C. Roberts, and A. Stancak, Shared and distinct functional networks for empathy and pain processing: a systematic review and meta-analysis of fMRI studies. Social cognitive and affective neuroscience, 2020. 15(7): p. 709-723.

50. Pasquini, L., F. Palhano-Fontes, and D.B. Araujo, Subacute effects of the psychedelic ayahuasca on the salience and default mode networks. Journal of Psychopharmacology, 2020. 34(6): p. 623635.

51. Valk, S.L., et al., Structural plasticity of the social brain: Differential change after socio-affective and cognitive mental training. Science Advances, 2017. 3(10): p. e1700489.

52. Kipps, C.M., et al., Understanding social dysfunction in the behavioural variant of frontotemporal dementia: the role of emotion and sarcasm processing. Brain, 2009. 132(3): p. 592-603.

53. Rankin, K.P., et al., Structural anatomy of empathy in neurodegenerative disease. Brain, 2006. 129(11): p. 2945-2956.

54. Margolis, A.E., et al., Salience network connectivity and social processing in children with nonverbal learning disability or autism spectrum disorder. Neuropsychology, 2019. 33(1): p. 135.

55. Sterzer, P., et al., A structural neural deficit in adolescents with conduct disorder and its association with lack of empathy. Neuroimage, 2007. 37(1): p. 335-342.

56. Mow, J.L., A. Gandhi, and D. Fulford, Imaging the "social brain" in schizophrenia: A systematic review of neuroimaging studies of social reward and punishment. Neuroscience \& Biobehavioral Reviews, 2020.

57. Ernst, J., et al., Interoceptive awareness enhances neural activity during empathy. Human Brain Mapping, 2013. 34(7): p. 1615-1624.

58. García-Cordero, I., et al., Feeling, learning from and being aware of inner states: interoceptive dimensions in neurodegeneration and stroke. Philosophical Transactions of the Royal Society B: Biological Sciences, 2016. 371(1708): p. 20160006.

59. Fletcher, P.D., et al., Pain and temperature processing in dementia: a clinical and neuroanatomical analysis. Brain, 2015. 138(11): p. 3360-3372.

60. Papagno, C., et al., Specific disgust processing in the left insula: new evidence from direct electrical stimulation. Neuropsychologia, 2016. 84: p. 29-35.

61. Meeter, L.H., et al., Imaging and fluid biomarkers in frontotemporal dementia. Nature Reviews Neurology, 2017. 13(7): p. 406.

62. Kurth, F., et al., A link between the systems: functional differentiation and integration within the human insula revealed by meta-analysis. Brain Structure and Function, 2010. 214(5-6): p. 519534.

63. Menon, V. and L.Q. Uddin, Saliency, switching, attention and control: a network model of insula function. Brain structure and function, 2010. 214(5-6): p. 655-667.

64. Ardila, A., B. Bernal, and M. Rosselli, Executive functions brain system: An activation likelihood estimation meta-analytic study. Archives of Clinical Neuropsychology, 2018. 33(4): p. 379-405.

65. Kiselica, A.M. and J.F. Benge, Quantitative and qualitative features of executive dysfunction in frontotemporal and Alzheimer's dementia. Applied Neuropsychology: Adult, 2019: p. 1-15.

66. Raczka, K.A., et al., Executive and behavioral deficits share common neural substrates in frontotemporal lobar degeneration-a pilot FDG-PET study. Psychiatry Research: Neuroimaging, 2010. 182(3): p. 274-280.

67. Roca, M., et al., Intelligence and executive functions in frontotemporal dementia. Neuropsychologia, 2013. 51(4): p. 725-730.

68. Radakovic, R., et al., Multidimensional Apathy in Behavioral Variant Frontotemporal Dementia, Primary Progressive Aphasia, and Alzheimer Disease. Journal of geriatric psychiatry and neurology, 2020: p. 0891988720924716. 
69. Walter, H., et al., Motivating forces of human actions: Neuroimaging reward and social interaction. Brain research bulletin, 2005. 67(5): p. 368-381.

70. Levy, R. and B. Dubois, Apathy and the functional anatomy of the prefrontal cortex-basal ganglia circuits. Cerebral cortex, 2006. 16(7): p. 916-928.

71. Wang, H., J. Zhang, and H. Jia, Separate neural systems value prosocial behaviors and reward: An ALE meta-analysis. Frontiers in human neuroscience, 2019. 13: p. 276.

72. Massimo, L., et al., Apathy in frontotemporal degeneration: neuroanatomical evidence of impaired goal-directed behavior. Frontiers in human neuroscience, 2015. 9: p. 611.

73. Fernández-Matarrubia, M., et al., Episodic memory dysfunction in behavioral variant frontotemporal dementia: a clinical and FDG-PET study. Journal of Alzheimer's Disease, 2017. 57(4): p. 1251-1264.

74. Bueno, A.P.A., et al., Papez circuit gray matter and episodic memory in amyotrophic lateral sclerosis and behavioural variant frontotemporal dementia. Brain Imaging and Behavior, 2020: p. 1-11.

75. Hornberger, M. and O. Piguet, Episodic memory in frontotemporal dementia: a critical review. Brain, 2012. 135(3): p. 678-692.

76. Balasa, M., et al., Diagnostic accuracy of behavioral variant frontotemporal dementia consortium criteria (FTDC) in a clinicopathological cohort. Neuropathology and applied neurobiology, 2015. 41(7): p. 882-892.

77. Beach, T.G., et al., Accuracy of the clinical diagnosis of Alzheimer disease at National Institute on Aging Alzheimer Disease Centers, 2005-2010. Journal of neuropathology and experimental neurology, 2012. 71(4): p. 266-273.

78. van der Zee, J., K. Sleegers, and C. Van Broeckhoven, Invited article: the Alzheimer diseasefrontotemporal lobar degeneration spectrum. Neurology, 2008. 71(15): p. 1191-1197.

79. Rohrer, J.D., Behavioural variant frontotemporal dementia-defining genetic and pathological subtypes. Journal of Molecular Neuroscience, 2011. 45(3): p. 583-588.

80. Bang, J., S. Spina, and B.L. Miller, Non-Alzheimer's dementia 1: Frontotemporal dementia. Lancet (London, England), 2015. 386(10004): p. 1672.

81. Salimi-Khorshidi, G., et al., Meta-analysis of neuroimaging data: a comparison of image-based and coordinate-based pooling of studies. Neuroimage, 2009. 45(3): p. 810-823. 


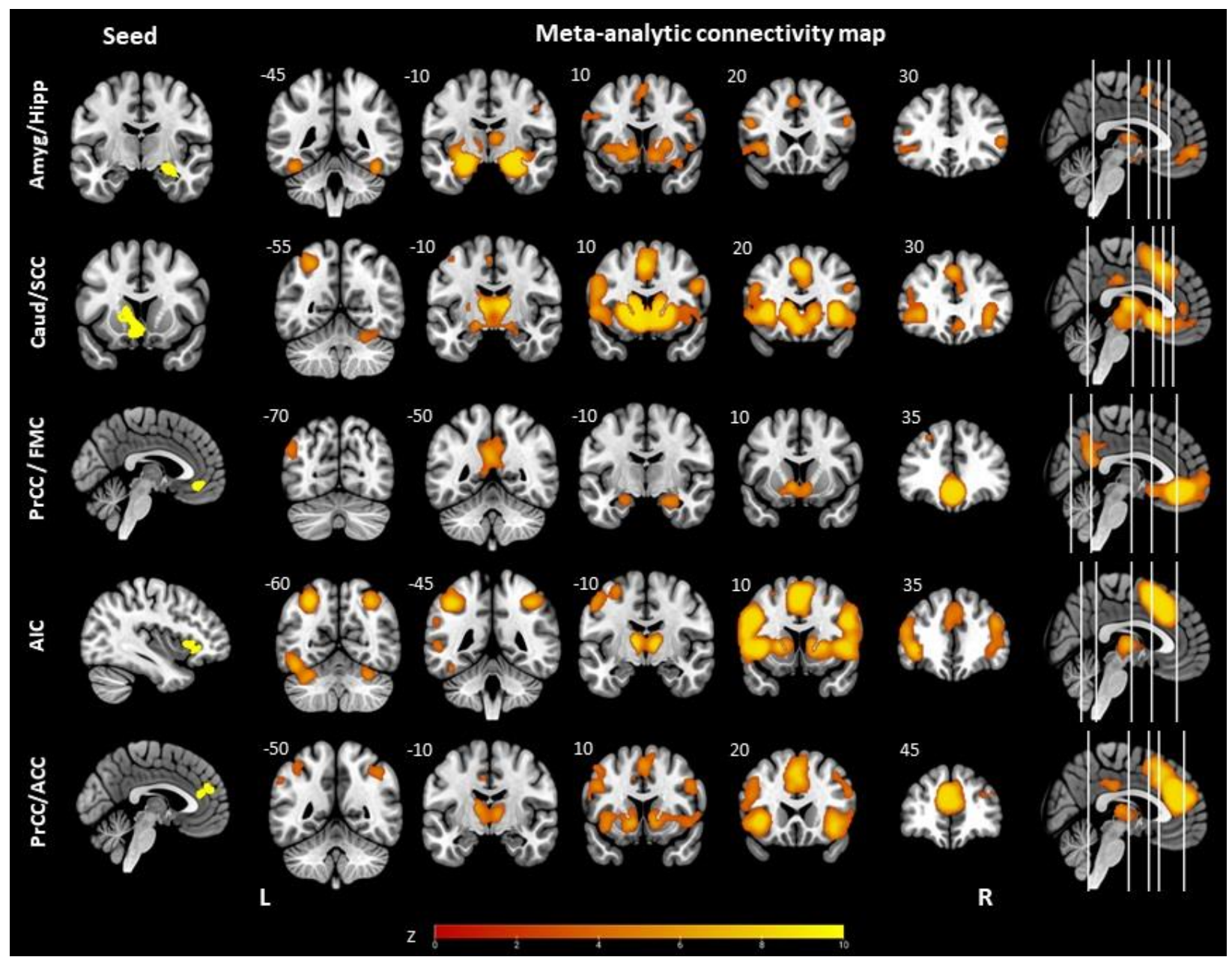

Supplementary Figure 1. Meta-analytic connectivity mapping of convergent regions in the all-effects activation likelihood estimation. The coordinates are in MNI space. Amyg: amygdala, Hipp: hippocampus, Caud: caudate nucleus, SCC: subcallosal cortex, PrCC: paracingulate cortex, FMC: frontomedial cortex, AIC: anterior insular cortex, ACC: anterior cingulate cortex. 


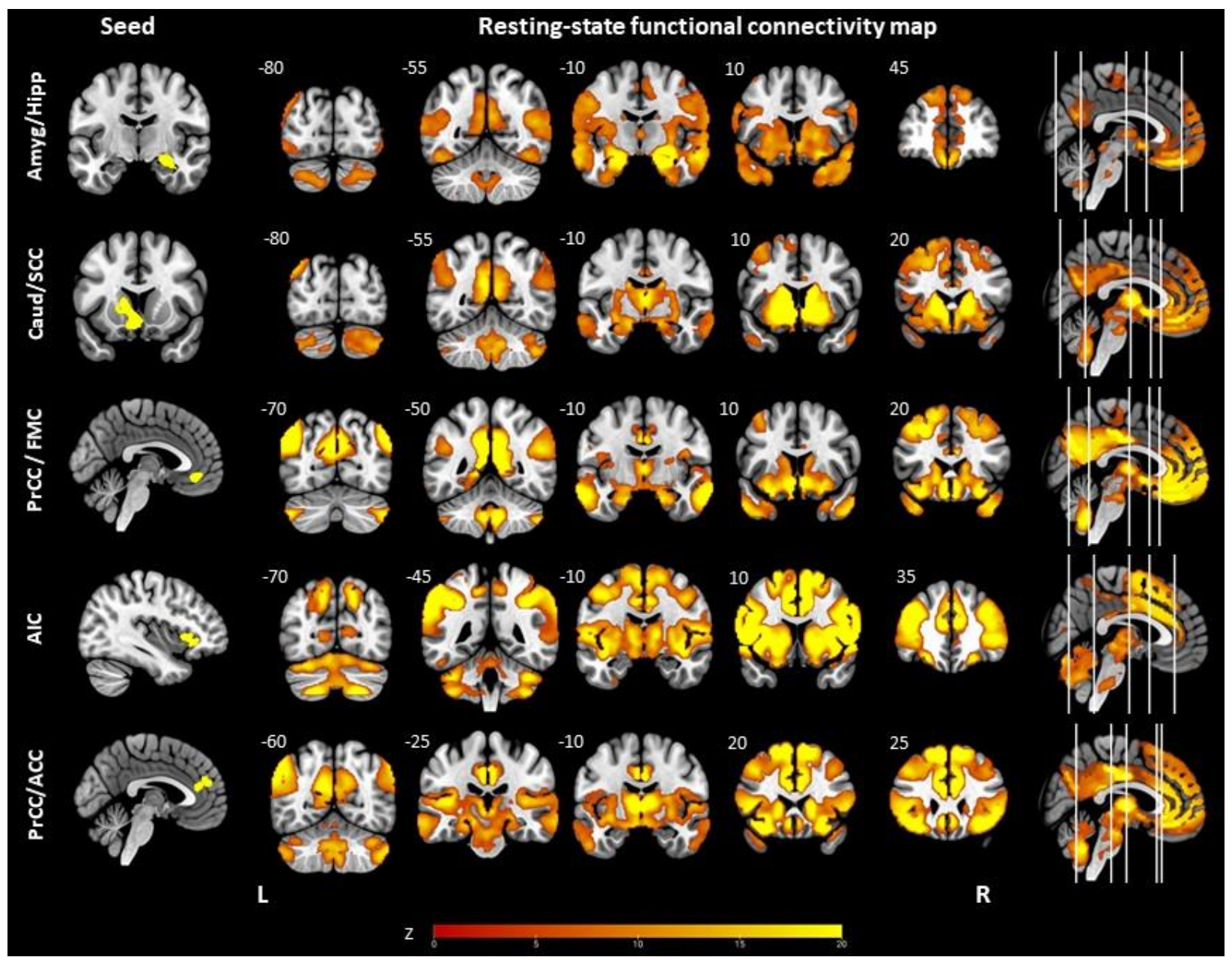

Supplementary Figure 2. Resting-state functional connectivity maps of convergent regions in the alleffects activation likelihood estimation. The coordinates are in MNI space. Amyg: amygdala, Hipp: hippocampus, Caud: caudate nucleus, SCC: subcallosal cortex, PrCC: paracingulate cortex, FMC: frontomedial cortex, AIC: anterior insular cortex, ACC: anterior cingulate cortex. 
Supplementary Table 1. Final studies included in the meta-analysis. (bvFTD: Behavioral variant of Frontotemporal dementia, MNI: Montreal Neurological Institute atlas, VBM: Voxelbased morphometry, PET: 18F-fluorodeoxyglucose positron emission tomography, rs-fMRI:

Resting-state functional magnetic resonance imaging, t-fMRI: Task-based functional magnetic resonance imaging, N/A: The required information was not available)

\begin{tabular}{|c|c|c|c|c|c|c|c|c|c|}
\hline \multirow[t]{2}{*}{ Ref. } & \multirow[t]{2}{*}{ Study } & \multicolumn{3}{|c|}{ Control } & \multicolumn{3}{|c|}{ bvFTD } & \multirow[t]{2}{*}{ Space } & \multirow{2}{*}{$\begin{array}{l}\text { Imaging } \\
\text { Method }\end{array}$} \\
\hline & & Numbers & $\begin{array}{l}\text { Mean } \\
\text { Age }\end{array}$ & $\begin{array}{l}\text { Mean } \\
\text { Years of } \\
\text { Education }\end{array}$ & Numbers & Mean Age & $\begin{array}{l}\text { Mean Years } \\
\text { of Education }\end{array}$ & & \\
\hline [1] & $\begin{array}{l}\text { Lee et al. } \\
2014\end{array}$ & 42 & 62.2 & 15.9 & $\begin{array}{l}\text { C9orf72 } \\
\text { carriers: } 14 \\
\text { Non-carrier: } \\
14\end{array}$ & $\begin{array}{l}\text { C9orf72 } \\
\text { carriers: } 58.3 \\
\text { Non-carrier: } \\
60.8\end{array}$ & $\begin{array}{l}\text { C9orf72 } \\
\text { carriers: } 14.9 \\
\text { Non-carrier: } \\
16.4\end{array}$ & MNI & VBM \\
\hline [2] & $\begin{array}{l}\text { Seeley et al. } \\
2008\end{array}$ & 45 & 68.3 & 16.4 & 15 & 65.9 & 14.7 & MNI & VBM \\
\hline [3] & $\begin{array}{l}\text { Mandelli et } \\
\text { al. } 2016\end{array}$ & 25 & 62.3 & 16.4 & 13 & 62.9 & 16.1 & MNI & VBM \\
\hline [4] & $\begin{array}{l}\text { Agosta et al. } \\
2009\end{array}$ & 56 & 66.57 & 16.58 & 31 & 58.53 & 15.95 & MNI & VBM \\
\hline [5] & $\begin{array}{l}\text { Chiong et al. } \\
2013\end{array}$ & 48 & 66 & 17.7 & 8 & 61.2 & 16.6 & MNI & VBM \\
\hline [6] & $\begin{array}{l}\text { Lagarde et al. } \\
2013\end{array}$ & 18 & 67.8 & 11.55 & 16 & 69.25 & 11.75 & MNI & VBM \\
\hline [7] & $\begin{array}{l}\text { Lagarde et al. } \\
2016\end{array}$ & 18 & 67.8 & 11.6 & 18 & 69.7 & 12 & MNI & VBM \\
\hline [8] & $\begin{array}{l}\text { Kumfor et al. } \\
2018\end{array}$ & 20 & 66.3 & 14.2 & 19 & 62.7 & 12.6 & MNI & VBM \\
\hline [9] & $\begin{array}{l}\text { Kamminga et } \\
\text { al. } 2015\end{array}$ & 14 & 65.8 & 11.2 & 13 & 60.5 & 11.3 & MNI & VBM \\
\hline [10] & $\begin{array}{l}\text { Kumfor et al. } \\
2014\end{array}$ & 10 & 71.3 & 14.9 & 13 & 66.5 & 11.1 & MNI & VBM \\
\hline [11] & $\begin{array}{l}\text { Kumfor et al. } \\
2015\end{array}$ & 10 & 68.6 & 14.8 & 10 & 62.5 & 12 & MNI & VBM \\
\hline [12] & $\begin{array}{l}\text { Kumfor et al. } \\
2018 \text { (a) }\end{array}$ & 28 & 63.9 & 12.5 & 55 & 62.9 & 11.8 & MNI & VBM \\
\hline [13] & $\begin{array}{l}\text { Kumfor et al. } \\
2017\end{array}$ & 21 & 64.8 & 13.7 & 22 & 65 & 11.1 & MNI & VBM \\
\hline [14] & $\begin{array}{l}\text { Hutchings et } \\
\text { al. } 2015\end{array}$ & 14 & 70.78 & 13.7 & 13 & 64.05 & 11.8 & MNI & VBM \\
\hline [15] & $\begin{array}{l}\text { Kumfor et al. } \\
2013\end{array}$ & 27 & 64.3 & 13.6 & 18 & 63.8 & 11.9 & MNI & VBM \\
\hline [16] & $\begin{array}{l}\text { Ahmed et al. } \\
2016\end{array}$ & 25 & 66 & 13.8 & 19 & 62 & 11.9 & MNI & VBM \\
\hline [17] & $\begin{array}{l}\text { Devenney et } \\
\text { al. } 2015\end{array}$ & 25 & N/A & 13.4 & $\begin{array}{l}\text { Probable } \\
\text { bvFTD: } 38 \\
\text { Possible } \\
\text { bvFTD: } 20\end{array}$ & N/A & $\begin{array}{l}\text { Probable } \\
\text { bvFTD: } 12.5 \\
\text { Possible } \\
\text { bvFTD: } 10.8\end{array}$ & MNI & VBM \\
\hline [18] & $\begin{array}{l}\text { Ahmed et al. } \\
2019\end{array}$ & 19 & 62.9 & N/A & 26 & 60.9 & N/A & MNI & VBM \\
\hline [19] & $\begin{array}{l}\text { Donnelly- } \\
\text { kehoe et al. } \\
2019\end{array}$ & 60 & 63.91 & 14.83 & 44 & 66.72 & 13.75 & MNI & VBM \\
\hline [20] & Kumfor et al. & 20 & 65.2 & 13.8 & 16 & 60.7 & 11.2 & MNI & VBM \\
\hline
\end{tabular}




\begin{tabular}{|c|c|c|c|c|c|c|c|c|c|}
\hline & 2018 (b) & & & & & & & & \\
\hline [21] & $\begin{array}{l}\text { Wei et al. } \\
2019\end{array}$ & 26 & 64.69 & 14.47 & 36 & 61.05 & 11.92 & MNI & VBM \\
\hline [22] & $\begin{array}{l}\text { Wilson et al. } \\
2019\end{array}$ & 22 & 66.1 & 14.2 & 18 & 62.5 & 11.8 & MNI & VBM \\
\hline [23] & $\begin{array}{l}\text { Irish et al. } \\
2013 \text { (a) }\end{array}$ & 10 & 69.2 & 12.7 & 10 & 62.8 & 11.8 & MNI & VBM \\
\hline [24] & $\begin{array}{l}\text { Irish et al. } \\
2013 \text { (b) }\end{array}$ & 19 & 68.1 & 13.1 & 19 & 63.6 & 12.6 & MNI & VBM \\
\hline [25] & $\begin{array}{l}\text { Irish et al. } \\
2014 \text { (a) }\end{array}$ & 14 & 72.2 & 11.9 & 11 & 62.4 & 11.9 & MNI & VBM \\
\hline [26] & $\begin{array}{l}\text { Irish et al. } \\
2016\end{array}$ & 20 & 67.1 & 13.3 & 15 & 63.5 & 11.6 & MNI & VBM \\
\hline [27] & $\begin{array}{l}\text { Dermody et } \\
\text { al. } 2016\end{array}$ & 12 & 69 & 13.6 & 12 & 63.2 & 11.6 & MNI & VBM \\
\hline [28] & $\begin{array}{l}\text { Dermody et } \\
\text { al. } 2015\end{array}$ & 22 & 68.2 & 14 & 24 & 63 & 12.4 & MNI & VBM \\
\hline [29] & $\begin{array}{l}\text { Hornberger } \\
\text { et al. } 2011\end{array}$ & 18 & 64.8 & 13.6 & 14 & 59.3 & 11.8 & MNI & VBM \\
\hline [30] & $\begin{array}{l}\text { Chen et al. } \\
2018 \text { (a) }\end{array}$ & 35 & 64.4 & 13.4 & 45 & 60.9 & 11.6 & MNI & VBM \\
\hline [31] & $\begin{array}{l}\text { Irish et al. } \\
2014 \text { (b) }\end{array}$ & 14 & 68 & 13.7 & 10 & 63.6 & 10.9 & MNI & VBM \\
\hline [32] & $\begin{array}{l}\text { O'Callaghan } \\
\text { et al. } 2019\end{array}$ & 24 & 66.3 & N/A & 35 & 61.4 & N/A & MNI & VBM \\
\hline [33] & Tu et al. 2015 & 23 & 68 & 13.3 & 24 & 64.7 & 11.8 & MNI & VBM \\
\hline [34] & $\begin{array}{l}\text { Baez et al. } \\
2017\end{array}$ & 22 & 62.5 & 14.2 & 16 & 65.8 & 14.8 & MNI & VBM \\
\hline [35] & $\begin{array}{l}\text { Baez et al. } \\
2016 \text { (a) }\end{array}$ & 23 & 62.69 & 15.65 & 26 & 66.08 & 15.24 & MNI & VBM \\
\hline [36] & $\begin{array}{l}\text { Baez et al. } \\
2016 \text { (b) }\end{array}$ & 19 & 60.42 & 15.21 & 21 & 63.8 & 14.23 & MNI & VBM \\
\hline [37] & $\begin{array}{l}\text { Cousins et al. } \\
2016\end{array}$ & 18 & 68.6 & 15.6 & 18 & 63.9 & 16.3 & MNI & VBM \\
\hline [38] & $\begin{array}{l}\text { McMillan et } \\
\text { al. } 2013\end{array}$ & 16 & 64.4 & 14.3 & 16 & 64.3 & 15.4 & MNI & VBM \\
\hline [39] & $\begin{array}{l}\text { Grossman et } \\
\text { al. } 2004\end{array}$ & 25 & N/A & N/A & 14 & 63.07 & 15.07 & MNI & VBM \\
\hline [40] & $\begin{array}{l}\text { Morgan et al. } \\
2011\end{array}$ & 40 & 65.9 & 15 & 8 & 64.9 & 14.6 & MNI & VBM \\
\hline [41] & $\begin{array}{l}\text { Libon et al. } \\
2009\end{array}$ & 16 & N/A & N/A & 51 & 61.31 & 15.58 & MNI & VBM \\
\hline [42] & $\begin{array}{l}\text { Nevler et al. } \\
2017\end{array}$ & 17 & 66 & 16.3 & 32 & 63 & 15.7 & MNI & VBM \\
\hline [43] & $\begin{array}{l}\text { Massimo et } \\
\text { al. } 2012\end{array}$ & 30 & N/A & N/A & 37 & 63.69 & 15.57 & Talairach & VBM \\
\hline [44] & $\begin{array}{l}\text { Ash et al. } \\
2016\end{array}$ & 27 & 67.1 & 14.9 & 32 & 63.7 & 15.7 & MNI & VBM \\
\hline [45] & $\begin{array}{l}\text { Healy et al. } \\
2015\end{array}$ & 35 & 70.2 & 15.54 & 9 & 65.3 & 15.75 & MNI & VBM \\
\hline [46] & $\begin{array}{l}\text { Kanda et al. } \\
2008\end{array}$ & 29 & 65.2 & N/A & 13 & 64.9 & N/A & MNI & $\begin{array}{l}\text { VBM } \\
\text { PET }\end{array}$ \\
\hline [47] & $\begin{array}{l}\text { Franceschi et } \\
\text { al. } 2005\end{array}$ & 24 & N/A & N/A & 6 & 61 & 12 & MNI & PET \\
\hline [48] & Salmon et al. & 58 & 60.1 & N/A & 29 & 61.5 & N/A & MNI & PET \\
\hline
\end{tabular}




\begin{tabular}{|c|c|c|c|c|c|c|c|c|c|}
\hline & 2003 & & & & & & & & \\
\hline [49] & $\begin{array}{l}\text { Tuovinen et } \\
\text { al. } 2017\end{array}$ & 25 & 59.6 & N/A & 21 & 60.7 & N/A & MNI & $\begin{array}{l}\text { VBM, } \\
\text { rs-fMRI }\end{array}$ \\
\hline [50] & $\begin{array}{l}\text { Rytty et al. } \\
2013\end{array}$ & 19 & 57.8 & N/A & 19 & 60.3 & N/A & MNI & $\begin{array}{l}\text { VBM, } \\
\text { rs-fMRI }\end{array}$ \\
\hline [51] & $\begin{array}{l}\text { Melloni et al. } \\
2016\end{array}$ & 22 & 68.31 & 13.06 & 26 & 67.96 & 13.92 & MNI & VBM \\
\hline [52] & $\begin{array}{l}\text { Fernandez- } \\
\text { Matarrubia } \\
\text { et al. } 2017\end{array}$ & 24 & 67.4 & 9.3 & $\begin{array}{l}\text { Amnesic- } \\
\text { FTD: } 26 \\
\text { Non- } \\
\text { Amnesic- } \\
\text { FTD: } 13\end{array}$ & $\begin{array}{l}\text { Amnesic- } \\
\text { FTD: } 71.3 \\
\text { Non- } \\
\text { Amnesic- } \\
\text { FTD: } 71.6\end{array}$ & $\begin{array}{l}\text { Amnesic- } \\
\text { FTD: } 8 \\
\text { Non- } \\
\text { Amnesic- } \\
\text { FTD: } 6.8\end{array}$ & MNI & PET \\
\hline [53] & $\begin{array}{l}\text { Matias et al. } \\
2018\end{array}$ & 19 & 64.89 & 13.89 & 19 & 71.05 & 8.21 & MNI & PET \\
\hline [54] & $\begin{array}{l}\text { Hafkemeijer } \\
\text { et al. } 2016\end{array}$ & 22 & 63.8 & 5.6 & 12 & 64.7 & 5 & MNI & VBM \\
\hline [55] & $\begin{array}{l}\text { Farb et al. } \\
2013\end{array}$ & 16 & 67.2 & 16.2 & 8 & 66.7 & 16 & MNI & VBM \\
\hline [56] & $\begin{array}{l}\text { Bertoux et al. } \\
2018\end{array}$ & 20 & 71.72 & 12.86 & 12 & 67.17 & 12.14 & MNI & VBM \\
\hline [57] & $\begin{array}{l}\text { Santmaria- } \\
\text { Garcia et al. } \\
2016\end{array}$ & 30 & 60.1 & 13.22 & 16 & 57 & 14.67 & MNI & VBM \\
\hline [58] & $\begin{array}{l}\text { Boccardi et } \\
\text { al. } 2005\end{array}$ & 26 & 69 & 8 & 9 & 62 & 6 & MNI & VBM \\
\hline [17] & $\begin{array}{l}\text { Devencchio et } \\
\text { al. } 2018\end{array}$ & $\begin{array}{l}\text { VBM: } \\
20 \\
\text { PET: } 48\end{array}$ & $\begin{array}{l}\text { VBM: } \\
49.8 \\
\text { PET: } \\
68.3\end{array}$ & $\begin{array}{l}\text { VBM: } \\
14.3 \\
\text { PET: } \\
10.3\end{array}$ & $\begin{array}{l}\text { VBM: } 21 \\
\text { PET: } 23\end{array}$ & $\begin{array}{l}\text { VBM: } 72.1 \\
\text { PET: } 71\end{array}$ & $\begin{array}{l}\text { VBM: } 10.8 \\
\text { PET: } 10.6\end{array}$ & MNI & $\begin{array}{l}\text { VBM, } \\
\text { PET }\end{array}$ \\
\hline [59] & $\begin{array}{l}\text { Jeong et al. } \\
2005\end{array}$ & 11 & 61.9 & N/A & 29 & 59.2 & N/A & MNI & PET \\
\hline [60] & $\begin{array}{l}\text { Diehl et al. } \\
2004\end{array}$ & 15 & 61.8 & N/A & 25 & 62.2 & N/A & Talairach & PET \\
\hline [61] & $\begin{array}{l}\text { Laws et al. } \\
2007\end{array}$ & 16 & 64.89 & N/A & $\begin{array}{l}\text { H1 } \\
\text { Homozygotes: } \\
31 \\
\text { H2 Carriers: } \\
10\end{array}$ & $\begin{array}{l}\text { H1 } \\
\text { Homozygotes: } \\
62.4 \\
\text { H2 Carriers: } \\
62.7\end{array}$ & $\begin{array}{l}\text { H1 } \\
\text { Homozygotes: } \\
\text { N/A } \\
\text { H2 Carriers: } \\
\text { N/A }\end{array}$ & Talairach & PET \\
\hline [62] & $\begin{array}{l}\text { Perneczhy et } \\
\text { al. } 2007\end{array}$ & 16 & 67.88 & 12.78 & 29 & 62.34 & 12.31 & Talairach & PET \\
\hline [63] & $\begin{array}{l}\text { Grimmer et } \\
\text { al. } 2004\end{array}$ & 15 & 61.8 & N/A & 10 & 59.9 & N/A & Talairach & PET \\
\hline [64] & $\begin{array}{l}\text { Peters et al. } \\
2006\end{array}$ & 23 & 64 & N/A & 23 & 63.5 & N/A & MNI & PET \\
\hline [65] & $\begin{array}{l}\text { Raczka et al. } \\
2010\end{array}$ & 9 & 59.3 & N/A & 7 & 60 & N/A & MNI & PET \\
\hline [66] & $\begin{array}{l}\text { Sheelakumari } \\
\text { et al. } 2019\end{array}$ & 20 & 62.8 & 15.63 & 25 & 63.68 & 17.03 & MNI & VBM \\
\hline [67] & $\begin{array}{l}\text { Caminiti et } \\
\text { al. } 2015\end{array}$ & 30 & 58.84 & 13.56 & 12 & 63.11 & 12.08 & MNI & rs-fMRI \\
\hline [68] & $\begin{array}{l}\text { Verfaillie et } \\
\text { al. } 2015\end{array}$ & 10 & 56 & N/A & 12 & 61 & N/A & MNI & PET \\
\hline [69] & $\begin{array}{l}\text { Meyer et al. } \\
2017\end{array}$ & $\begin{array}{l}\text { First } \\
\text { group: } \\
19\end{array}$ & $\begin{array}{l}\text { First } \\
\text { group: } \\
63.1\end{array}$ & $\begin{array}{l}\text { First } \\
\text { group: } \\
\text { N/A }\end{array}$ & $\begin{array}{l}\text { First group: } \\
19 \\
\text { Second group: }\end{array}$ & $\begin{array}{l}\text { First group: } \\
61.5 \\
\text { Second group: }\end{array}$ & $\begin{array}{l}\text { First group: } \\
\text { N/A } \\
\text { Second group: }\end{array}$ & MNI & VBM \\
\hline
\end{tabular}




\begin{tabular}{|l|l|l|l|l|l|l|l|l|l|}
\hline & & $\begin{array}{l}\text { Second } \\
\text { group: } \\
52\end{array}$ & $\begin{array}{l}\text { Second } \\
\text { group: } \\
63.1\end{array}$ & $\begin{array}{l}\text { Second } \\
\text { group: } \\
\text { N/A }\end{array}$ & 52 & 61.5 & N/A & & \\
\hline [70] & $\begin{array}{l}\text { Garcia- } \\
\text { Cordero et al. } \\
\mathbf{2 0 1 5}\end{array}$ & 14 & 57.21 & 16.14 & 11 & 64.81 & 14.45 & MNI & VBM \\
\hline [71] & $\begin{array}{l}\text { Kipps et al. } \\
\text { 2009 }\end{array}$ & 16 & 66.4 & N/A & 11 & 62.1 & N/A & MNI & VBM \\
\hline [72] & $\begin{array}{l}\text { Morbelli et } \\
\text { al. 2016 }\end{array}$ & 20 & 64.4 & 13.4 & 25 & 60.9 & 11.6 & Talairach & $\begin{array}{l}\text { VBM, } \\
\text { PET }\end{array}$ \\
\hline [73] & $\begin{array}{l}\text { Augustus et } \\
\text { al. 2015 }\end{array}$ & 11 & 64 & N/A & 15 & 64 & N/A & MNI & t-fMRI \\
\hline
\end{tabular}


Supplementary Table 2. The excluded studies and the reason for their exclusion. (Not bv: The imaging analysis was not performed on the behavioral variant of frontotemporal dementia patients/other types of demented patients were included, Data Not Reported: The coordinates that were needed to perform the meta-analysis were not provided in the article or by the corresponding author when contacted, ROI: The study was excluded for performing analysis on region(s) of interest or a seed was used in functional connectivity studies, Not Case Control: The between-group contrasts was not performed between healthy controls and behavioral variant frontotemporal dementia patients)

\section{Title of The Article}

(18)F-flortaucipir (AV-1451) tau PET in frontotemporal dementia syndromes

[(18)F]-AV-1451 tau PET imaging in Alzheimer's disease and suspected non-AD tauopathies using a late acquisition time window

[18F]FDG-PET in a case of right temporal lobe variant of frontotemporal dementia

11C-DTBZ and 18F-FDG PET measures in differentiating dementias.

18F-florbetapir PET in patients with frontotemporal dementia and Alzheimer disease.

A Case of Crossed Logopenic Primary Progressive Aphasia in a Dextral Patient with Underlying

Frontotemporal Dementia

A critical role for the hippocampus in the valuation of imagined outcomes.

A multivariate metabolic imaging marker for behavioral variant frontotemporal dementia

A neural network underlying intentional emotional facial expression in neurodegenerative disease.

A non-DM1, non-DM2 multisystem myotonic disorder with frontotemporal dementia: phenotype and

suggestive mapping of the DM3 locus to chromosome 15q21-24.

A physiological signature of sound meaning in dementia.

A tensor based morphometry study of longitudinal gray matter contraction in FTD.

A visual MRI atrophy rating scale for the amyotrophic lateral sclerosis-frontotemporal dementia continuum.

A volumetric magnetic resonance imaging study of the amygdala in frontotemporal lobar degeneration and Alzheimer's disease.

Active lifestyles moderate clinical outcomes in autosomal dominant frontotemporal degeneration

Affective mentalizing and brain activity at rest in the behavioral variant of frontotemporal dementia.

Agreement among neuropsychological and behavioral data and PiB findings in diagnosing

Frontotemporal Dementia.

Alterations of the sense of "humanness" in right hemisphere predominant frontotemporal dementia patients.

Altered functional connectivity in asymptomatic MAPT subjects: a comparison to bvFTD.

Altered speech-related cortical network in frontotemporal dementia

Alzheimer Disease and Behavioral Variant Frontotemporal Dementia: Automatic Classification Based on Cortical Atrophy for Single-Subject Diagnosis.

Alzheimer's disease and frontotemporal dementia exhibit distinct atrophy-behavior correlates: a computer-assisted imaging study.

Amygdala subnuclei are differentially affected in the different genetic and pathological forms of

frontotemporal dementia

Amygdaloid atrophy in frontotemporal dementia and Alzheimer's disease.

Reason for Exclusion

18 F-flortaucipir and 11C-PiB

PET Imaging

$[18 \mathrm{~F}]-\mathrm{AV}-1451$ tau PET Imaging

Case Report

Not bv

18F-florbetapir PET Imaging

Case Report

Data Not Reported

Data Not Reported

Data Not Reported

Not bv

ROI

Tensor Based Morphometry

ROI

ROI

Not bv

ROI

Not bv

Irrelevant

ROI

ROI

Irrelevant

Not bv

ROI

ROI

ROI

11C-PiB PET Imaging

Amyloid Imaging With 11C-PIB in Patients With Cognitive Impairment in a Clinical Setting: A Visual and Semiquantitative Analysis.

Amyloid in dementia associated with familial FTLD: not an innocent bystander.
Not Case Control 


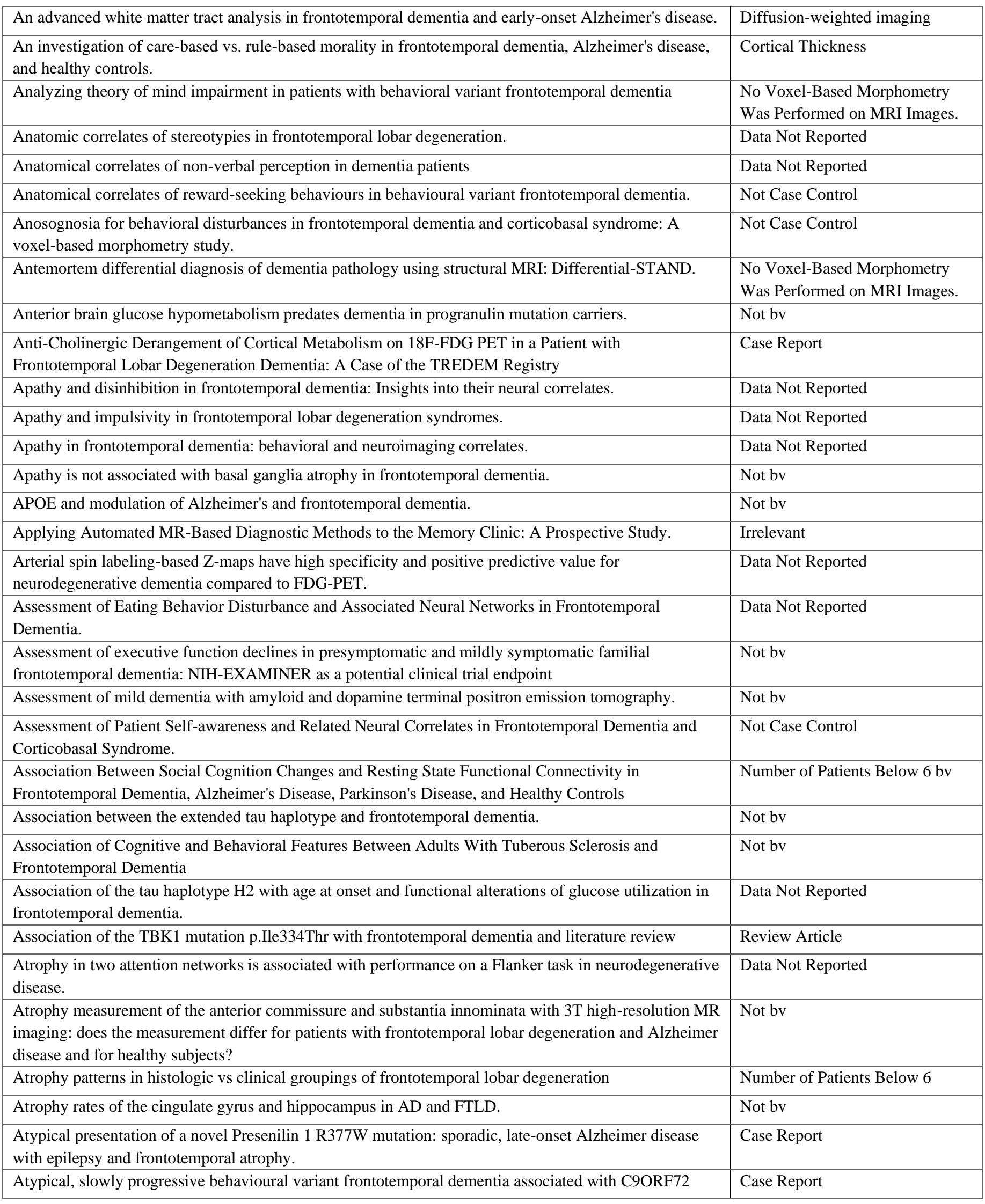




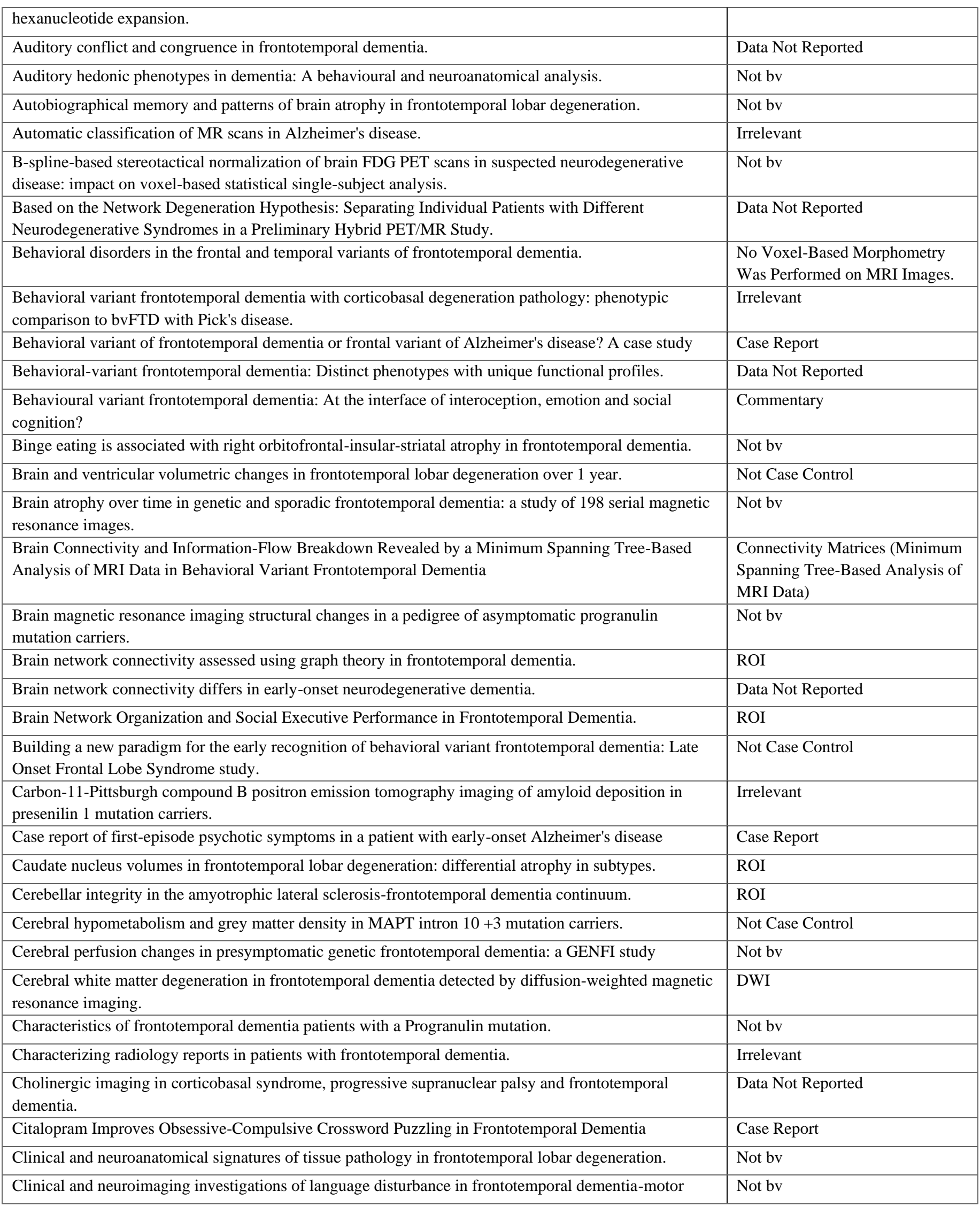




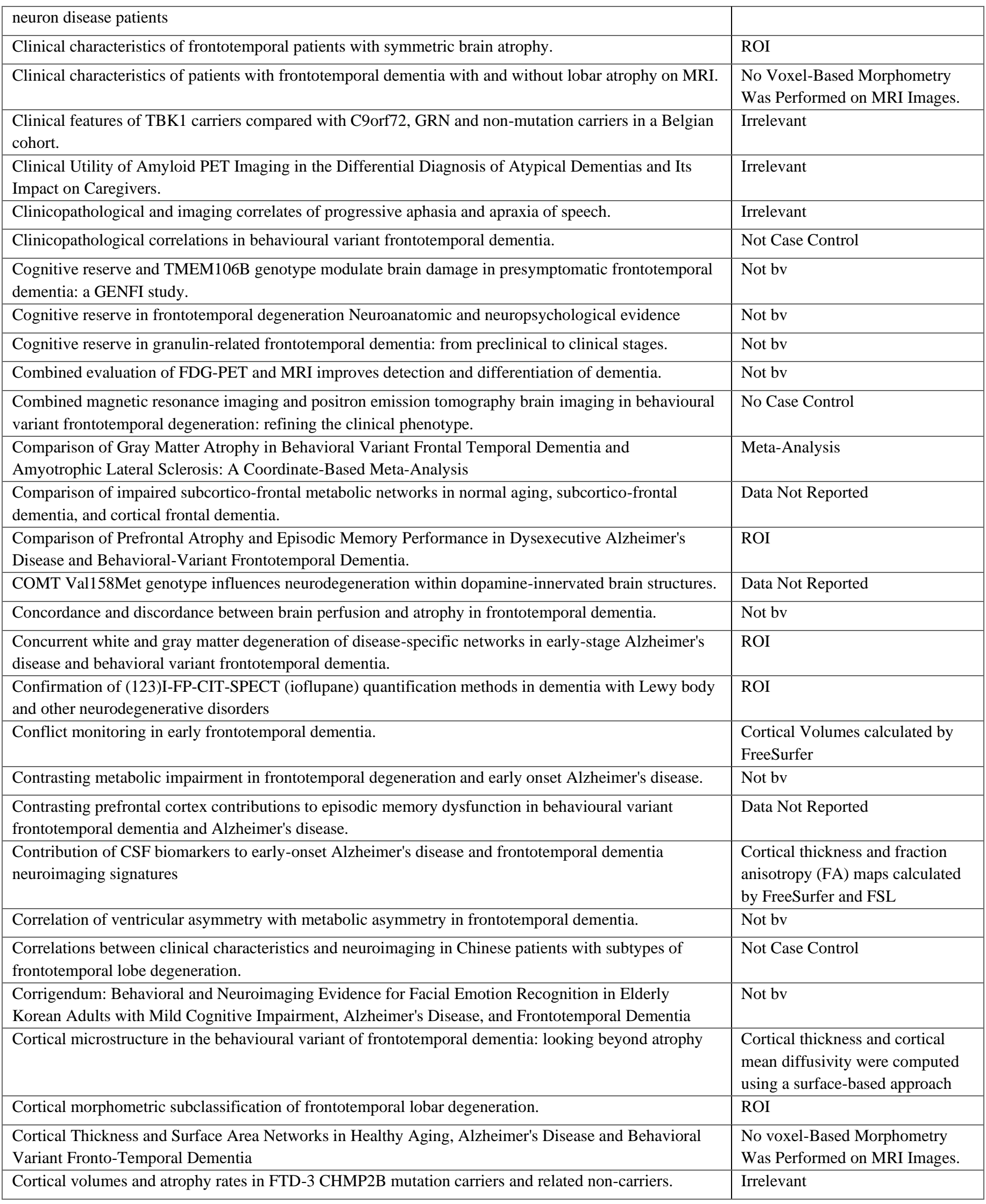




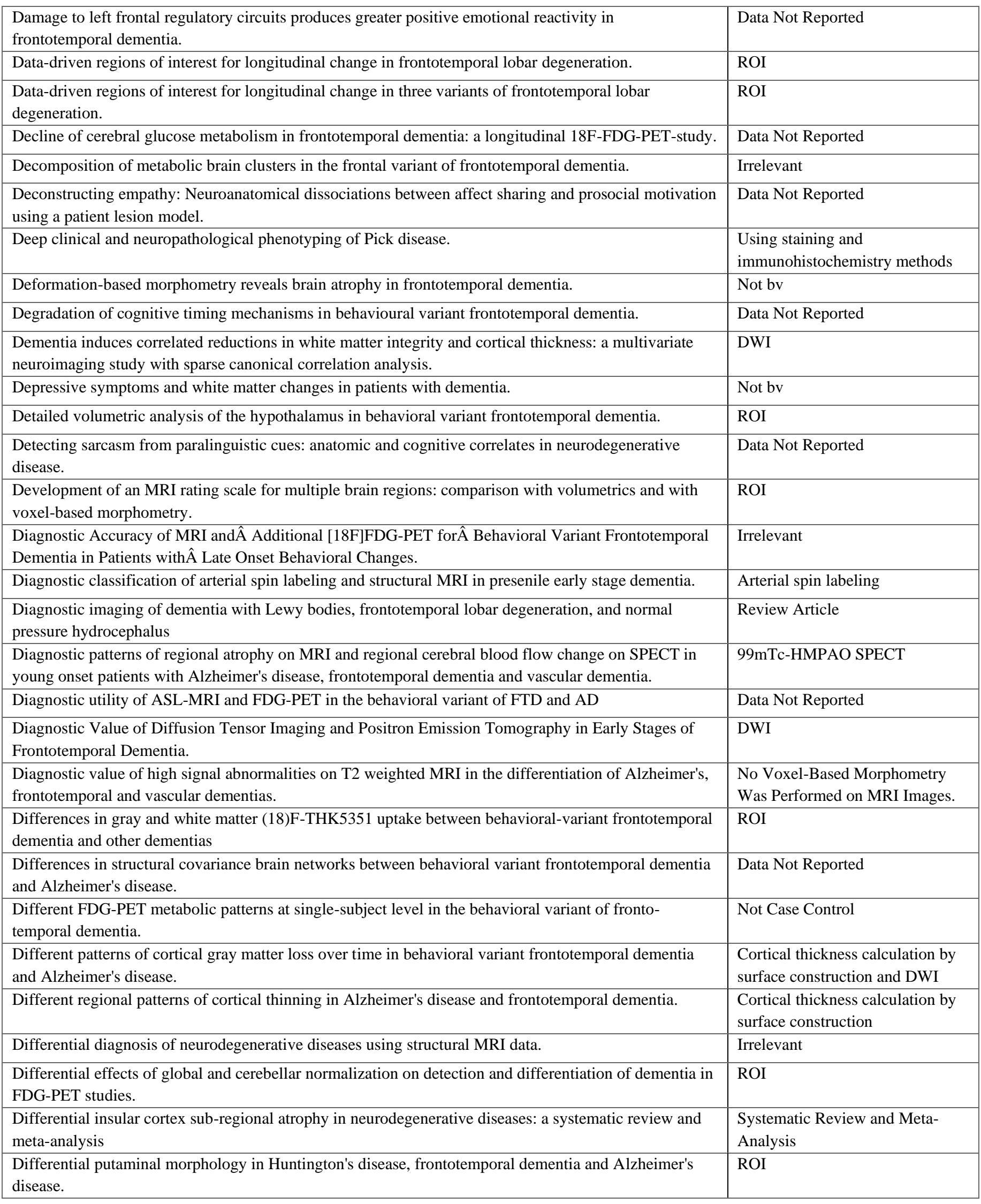


Differential topography of Alzheimer's disease and frontotemporal dementia: an MRI volumetric analysis from Sunnybrook dementia study.

Differentiating primary progressive aphasias in a brief sample of connected speech.

Diffusion tensor tractography versus volumetric imaging in the diagnosis of behavioral variant frontotemporal dementia.

Direct prospective comparison of (18)F-FDG PET and arterial spin labelling MR using simultaneous

PET/MR in patients referred for diagnosis of dementia

Discrete Neural Correlates for the Recognition of Negative Emotions: Insights from Frontotemporal

Dementia.

Discriminant validity and neuroanatomical correlates of rule monitoring in frontotemporal dementia and Alzheimer's disease.

Disease state fingerprint in frontotemporal degeneration with reference to Alzheimer's disease and mild cognitive impairment.

Disease-specific patterns of cortical and subcortical degeneration in a longitudinal study of Alzheimer's disease and behavioural-variant frontotemporal dementia.

Disentangling Heterogeneity in Alzheimer's Disease and Related Dementias Using Data-Driven Methods

Disrupted rich club network in behavioral variant frontotemporal dementia and early-onset Alzheimer's disease.

Dissociating behavioral disorders in early dementia-An FDG-PET study.

Dissociating memory networks in early Alzheimer's disease and frontotemporal lobar degeneration - a combined study of hypometabolism and atrophy.

Dissociation of Structural and Functional Integrities of the Motor System in Amyotrophic Lateral

Sclerosis and Behavioral-Variant Frontotemporal Dementia.

Distinct anatomical subtypes of the behavioural variant of frontotemporal dementia: a cluster analysis study.

Distinct antemortem profiles in patients with pathologically defined frontotemporal dementia.

Distinct MRI atrophy patterns in autopsy-proven Alzheimer's disease and frontotemporal lobar degeneration.

Distinct neuroanatomical substrates and cognitive mechanisms of figure copy performance in

Alzheimer's disease and behavioral variant frontotemporal dementia.

Distinct profiles of brain atrophy in frontotemporal lobar degeneration caused by progranulin and tau mutations.

Distinct Subtypes of Behavioral Variant Frontotemporal Dementia Based on Patterns of Network

Degeneration.

Divergent network connectivity changes in behavioural variant frontotemporal dementia and Alzheimer's disease.

Does TDP-43 type confer a distinct pattern of atrophy in frontotemporal lobar degeneration?

Dominant hemisphere lateralization of cortical parasympathetic control as revealed by frontotemporal dementia.

Dual-phase [18F]florbetapir in frontotemporal dementia

Dyslexia susceptibility genes influence brain atrophy in frontotemporal dementia.

Early 11C-PIB frames and 18F-FDG PET measures are comparable: a study validated in a cohort of AD and FTLD patients.

Early-stage differentiation between presenile Alzheimer's disease and frontotemporal dementia using arterial spin labeling MRI.

Eating and hypothalamus changes in behavioral-variant frontotemporal dementia.

Eating behavior in frontotemporal dementia: Peripheral hormones vs hypothalamic pathology.

Education modulates brain maintenance in presymptomatic frontotemporal dementia

Energy expenditure in frontotemporal dementia: a behavioural and imaging study. paper and abstract not available

Irrelevant

ROI

Number of Patients Below 6

Data Not Reported

ROI

Irrelevant

Cortical thickness calculation by surface construction

Review Article

DWI

Number of Patients Below 6

Number of Patients Below 6

ROI

ROI

Not bv

Not bv

Cortical thickness calculation by surface construction

Not bv

ROI

ROI

Not bv

ROI

[18F]florbetapir PET scan

Not Case Control

ROI

Arterial spin labeling MRI

ROI

ROI

Not bv

ROI 


\begin{tabular}{|c|c|}
\hline $\begin{array}{l}\text { Enhanced positive emotional reactivity undermines empathy in behavioral variant frontotemporal } \\
\text { dementia }\end{array}$ & Data Not Reported \\
\hline Episodic memory and regional atrophy in frontotemporal lobar degeneration. & Not bv \\
\hline $\begin{array}{l}\text { Executive Abilities as Reflected by Clock Hand Placement: Frontotemporal Dementia Versus Early- } \\
\text { Onset Alzheimer Disease. }\end{array}$ & Tensor Based Morphometry \\
\hline $\begin{array}{l}\text { Executive and behavioral deficits share common neural substrates in frontotemporal lobar degeneration - } \\
\text { a pilot FDG-PET study. }\end{array}$ & Not bv \\
\hline Executive deficits are related to the inferior frontal junction in early dementia. & Not Case Control \\
\hline Executive dysfunction in frontotemporal dementia and corticobasal syndrome. & Data Not Reported \\
\hline $\begin{array}{l}\text { Exploring quantitative group-wise differentiation of Alzheimer's disease and behavioural variant } \\
\text { frontotemporal dementia using tract-specific microstructural white matter and functional connectivity } \\
\text { measures at multiple time points }\end{array}$ & ROI \\
\hline Extrapyramidal symptoms in Frontotemporal Dementia: prevalence and clinical correlations. & Not bv \\
\hline $\begin{array}{l}\text { Face shape and face identity processing in behavioral variant fronto-temporal dementia: A specific } \\
\text { deficit for familiarity and name recognition of famous faces. }\end{array}$ & Data Not Reported \\
\hline Fair play: social norm compliance failures in behavioural variant frontotemporal dementia. & ROI \\
\hline $\begin{array}{l}\text { False Recognition in Behavioral Variant Frontotemporal Dementia and Alzheimer's Disease- } \\
\text { Disinhibition or Amnesia? }\end{array}$ & ROI \\
\hline FDG-PET improves accuracy in distinguishing frontotemporal dementia and Alzheimer's disease. & Not bv \\
\hline Fear conditioning in frontotemporal lobar degeneration and Alzheimer's disease. & Not bv \\
\hline First symptoms--frontotemporal dementia versus Alzheimer's disease. & Not Case Control \\
\hline Flavour identification in frontotemporal lobar degeneration. & Data Not Reported \\
\hline $\begin{array}{l}\text { Fluorodeoxyglucose positron emission tomography in semantic dementia after 6â€\%omonths of } \\
\text { memantine: an open-label pilot study. }\end{array}$ & Irrelevant \\
\hline $\begin{array}{l}\text { Fluorodeoxyglucose-positron emission tomography in the differential diagnosis of early-onset dementia: } \\
\text { a prospective, community-based study. }\end{array}$ & Irrelevant \\
\hline Frequency of ubiquitin and FUS-positive, TDP-43-negative frontotemporal lobar degeneration. & Not bv \\
\hline $\begin{array}{l}\text { Frontal asymmetry in behavioral variant frontotemporal dementia: clinicoimaging and pathogenetic } \\
\text { correlates. }\end{array}$ & Data Not Reported \\
\hline $\begin{array}{l}\text { Frontal network syndrome testing: clinical tests and positron emission tomography brain imaging help } \\
\text { distinguish the } 3 \text { most common dementia subtypes. }\end{array}$ & Not Case Control \\
\hline $\begin{array}{l}\text { Frontal variant of Alzheimer's disease with asymmetric presentation mimicking frontotemporal } \\
\text { dementia: Case report and literature review }\end{array}$ & Case Report \\
\hline $\begin{array}{l}\text { Fronto-striatal atrophy correlates of inhibitory dysfunction in Parkinson's disease versus behavioural } \\
\text { variant frontotemporal dementia. }\end{array}$ & ROI \\
\hline Frontotemporal asymmetry in socioemotional behavior: A pilot study in frontotemporal dementia & $\begin{array}{l}\text { Cortical Volumes calculated by } \\
\text { FreeSurfer }\end{array}$ \\
\hline Frontotemporal brain sagging syndrome: an SIH-like presentation mimicking FTD. & Irrelevant \\
\hline Frontotemporal dementia and its variants: What to look for & Under 6 No. of Patients \\
\hline $\begin{array}{l}\text { Frontotemporal dementia and language networks: cortical thickness reduction is driven by dyslexia } \\
\text { susceptibility genes. }\end{array}$ & Not Case Control \\
\hline Frontotemporal dementia associated with the C9ORF72 mutation: a unique clinical profile. & Not bv \\
\hline $\begin{array}{l}\text { Frontotemporal dementia can be distinguished from Alzheimer's disease and subcortical white matter } \\
\text { dementia by an anterior-to-posterior rCBF-SPET ratio. }\end{array}$ & $\begin{array}{l}\text { Single-photon emission } \\
\text { tomography (SPET; }(99 \mathrm{~m}) \mathrm{Tc}- \\
\text { HMPAO) }\end{array}$ \\
\hline Frontotemporal dementia due to C9ORF72 mutations: clinical and imaging features. & Not Case Control \\
\hline Frontotemporal dementia selectively impairs transitive reasoning about familiar spatial environments. & Data Not Reported \\
\hline $\begin{array}{l}\text { Frontotemporal dementia with the C9ORF72 hexanucleotide repeat expansion: clinical, neuroanatomical } \\
\text { and neuropathological features. }\end{array}$ & Not bv \\
\hline
\end{tabular}




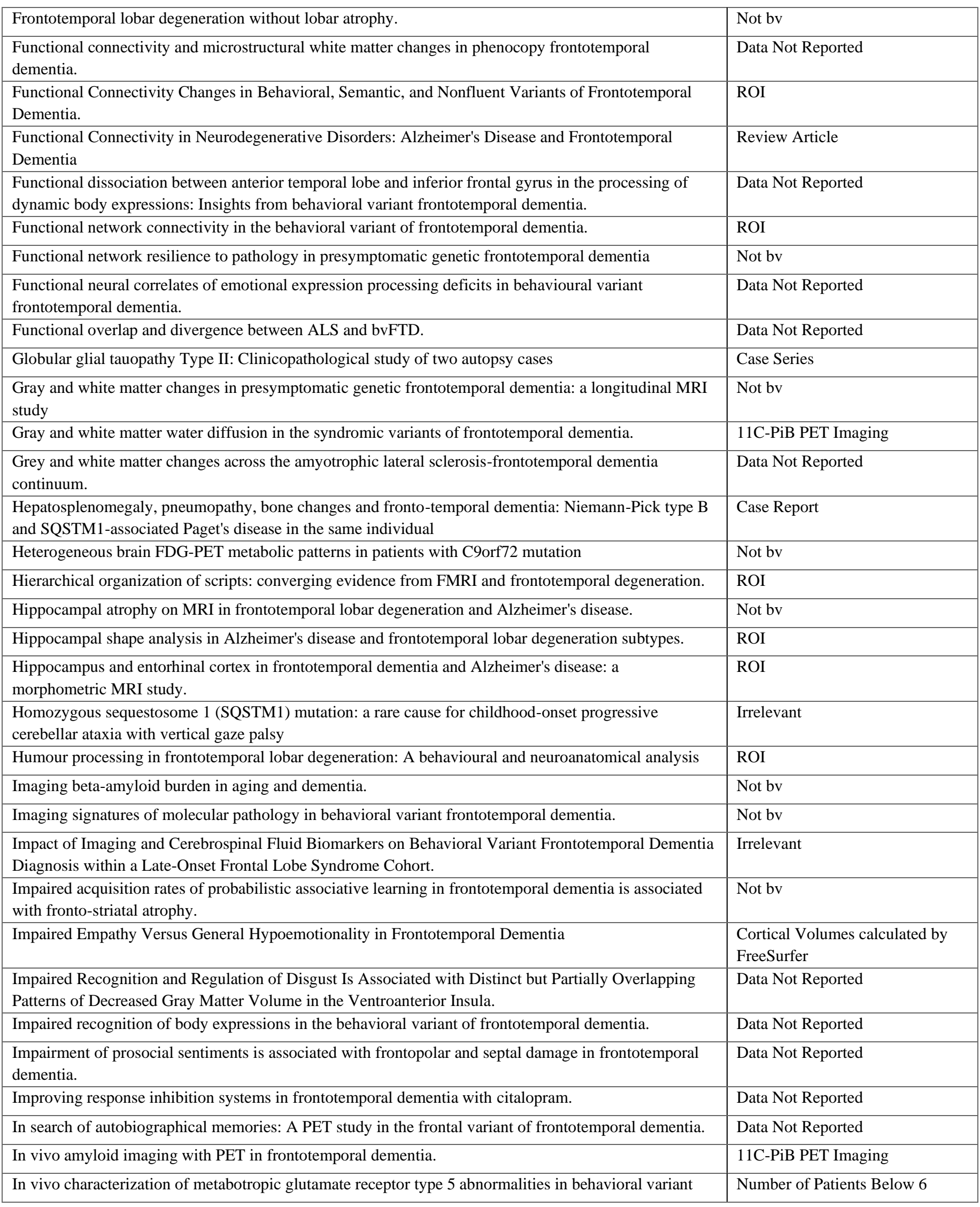


FTD.

In vivo evidence of cortical amyloid deposition in the adult form of Niemann Pick type $\mathrm{C}$

In vivo staging of frontotemporal lobar degeneration TDP-43 type C pathology

Individual patient diagnosis of $\mathrm{AD}$ and FTD via high-dimensional pattern classification of MRI.

Individualized atrophy scores predict dementia onset in familial frontotemporal lobar degeneration

Inheritance of the ApoE epsilon4 allele increases the rate of brain atrophy in dementia patients.

Insight in frontotemporal dementia: conceptual analysis and empirical evaluation of the consensus

criterion "loss of insight" in frontotemporal dementia.

Investigation of (11)C-PiB equivocal PET findings.

Is hippocampal volume a good marker to differentiate Alzheimer's disease from frontotemporal dementia?

Joint assessment of structural, perfusion, and diffusion MRI in Alzheimer's disease and frontotemporal

dementia

Joint assessment of white matter integrity, cortical and subcortical atrophy to distinguish AD from

behavioral variant FTD: A two-center study.

Lateral Temporal Lobe: An Early Imaging Marker of the Presymptomatic GRN Disease?

Lateralization on neuroimaging does not differentiate frontotemporal lobar degeneration from

Alzheimer's disease.

Left frontal lobe contributions to concept formation: a quantitative MRI study of performance on the

Delis-Kaplan Executive Function System Sorting Test.

Lessons of the month 3: Spontaneous resolution of frontotemporal brain sagging syndrome

Longitudinal ((18)F)AV-1451 PET imaging in a patient with frontotemporal dementia due to a Q351R

MAPT mutation

Longitudinal (18)F-FDG PET and MRI Reveal Evolving Imaging Pathology That Corresponds to

Disease Progression in a Patient With ALS-FTD

Longitudinal Amyloid- $\dot{\tau}^{2}$ PET in Atypical Alzheimer's Disease and Frontotemporal Lobar Degeneration

Longitudinal analysis of brain atrophy in Alzheimer's disease and frontotemporal dementia

Longitudinal grey and white matter changes in frontotemporal dementia and Alzheimer's disease.

Longitudinal Memory Profiles in Behavioral-Variant Frontotemporal Dementia and Alzheimer's

Disease.

Longitudinal neuroimaging and neuropsychological profiles of frontotemporal dementia with C9ORF72

expansions.

Longitudinal patterns of regional change on volumetric MRI in frontotemporal lobar degeneration.

Longitudinal rates of lobar atrophy in frontotemporal dementia, semantic dementia, and Alzheimer's

disease.

Longitudinal white matter changes in frontotemporal dementia subtypes.

Looking for Measures of Disease Severity in the Frontotemporal Dementia Continuum.

Looking for Neuroimaging Markers in Frontotemporal Lobar Degeneration Clinical Trials: A Multi-

Voxel Pattern Analysis Study in Granulin Disease.

Loss of frontal fMRI activation in early frontotemporal dementia compared to early AD.

Loss of insight and functional neuroimaging in frontotemporal dementia.

Lost and forgotten? Orientation versus memory in Alzheimer's disease and frontotemporal dementia.

Magnetic resonance imaging and histopathology in dementia, clinically of frontotemporal type.

Magnetic resonance imaging correlates of set shifting.

Magnetic resonance imaging in frontotemporal dementia shows subcortical atrophy.

Magnetic resonance imaging signatures of tissue pathology in frontotemporal dementia.

18F- Florbetaben PET Scan

Not bv

Irrelevant

Not bv

Not bv

Not bv

ROI

ROI

Data Not Reported

Data Not Reported

Not bv

Not bv

ROI

Case Report

Case Report

Case Report

11C-PiB PET Imaging

Case Series

ROI

11C-PiB PET Imaging

Not bv

Not bv

No voxel-Based Morphometry

Was Performed on MRI Images.

Data Not Reported

Not Case Control

Not bv

Not Case Control

Not Case Control

Data Not Reported

Not bv

ROI

ROI

Not bv 


\begin{tabular}{|c|c|}
\hline $\begin{array}{l}\text { Male gender is associated with greater cerebral hypometabolism in frontotemporal dementia: evidence } \\
\text { for sex-related cognitive reserve. }\end{array}$ & Not Case Control \\
\hline $\begin{array}{l}\text { Measurements of the amygdala and hippocampus in pathologically confirmed Alzheimer disease and } \\
\text { frontotemporal lobar degeneration. }\end{array}$ & Not bv \\
\hline Measuring disease progression in frontotemporal lobar degeneration: a clinical and MRI study. & Irrelevant \\
\hline Measuring network disruption in neurodegenerative diseases: New approaches using signal analysis & Review Article \\
\hline Medial temporal lobe metabolic impairment in dementia associated with motor neuron disease. & Not bv \\
\hline $\begin{array}{l}\text { Medial versus lateral frontal lobe contributions to voluntary saccade control as revealed by the study of } \\
\text { patients with frontal lobe degeneration. }\end{array}$ & Not bv \\
\hline $\begin{array}{l}\text { Memory consolidation and the hippocampus: further evidence from studies of autobiographical memory } \\
\text { in semantic dementia and frontal variant frontotemporal dementia. }\end{array}$ & Number of Patients Below 6 \\
\hline Memory profiles in pathology or biomarker confirmed Alzheimer disease and frontotemporal dementia. & Number of Patients Below 6 \\
\hline Mentalising music in frontotemporal dementia. & ROI \\
\hline Metabolic connectivity for differential diagnosis of dementing disorders. & Not bv \\
\hline Mirror neurons and their relationship with neurodegenerative disorders & Not bv \\
\hline $\begin{array}{l}\text { Money for nothing - Atrophy correlates of gambling decision making in behavioural variant } \\
\text { frontotemporal dementia and Alzheimer's disease }\end{array}$ & Data Not Reported \\
\hline $\begin{array}{l}\text { Moral processing deficit in behavioral variant frontotemporal dementia is associated with facial emotion } \\
\text { recognition and brain changes in default mode and salience network areas }\end{array}$ & Data Not Reported \\
\hline $\begin{array}{l}\text { More atrophy of deep gray matter structures in frontotemporal dementia compared to Alzheimer's } \\
\text { disease. }\end{array}$ & Not bv \\
\hline Motor speech signature of behavioral variant frontotemporal dementia: Refining the phenotype. & ROI \\
\hline $\begin{array}{l}\text { MR analysis of the substantia innominata in normal aging, Alzheimer disease, and other types of } \\
\text { dementia. }\end{array}$ & Not bv \\
\hline $\begin{array}{l}\text { MRI correlates of protein deposition and disease severity in postmortem frontotemporal lobar } \\
\text { degeneration. }\end{array}$ & Data Not Reported \\
\hline MRI of the Swallow Tail Sign: A Useful Marker in the Diagnosis of Lewy Body Dementia? & Irrelevant \\
\hline $\begin{array}{l}\text { MRI patterns of atrophy and hypoperfusion associations across brain regions in frontotemporal } \\
\text { dementia. }\end{array}$ & $\begin{array}{l}\text { Deformation-based morphometry } \\
\text { (DBM) }\end{array}$ \\
\hline $\begin{array}{l}\text { MRI signatures of brain macrostructural atrophy and microstructural degradation in frontotemporal lobar } \\
\text { degeneration subtypes. }\end{array}$ & Data Not Reported \\
\hline MRI signatures of the frontotemporal lobar degeneration continuum. & Data Not Reported \\
\hline MRI visual rating scales in the diagnosis of dementia: evaluation in 184 post-mortem confirmed cases. & Not bv \\
\hline $\begin{array}{l}\text { Multicenter standardized 18F-FDG PET diagnosis of mild cognitive impairment, Alzheimer's disease, } \\
\text { and other dementias. }\end{array}$ & Not bv \\
\hline $\begin{array}{l}\text { Multimodal FMRI resting-state functional connectivity in granulin mutations: the case of fronto-parietal } \\
\text { dementia. }\end{array}$ & Not bv \\
\hline $\begin{array}{l}\text { Multimodal MRI of grey matter, white matter, and functional connectivity in cognitively healthy } \\
\text { mutation carriers at risk for frontotemporal dementia and Alzheimer's disease }\end{array}$ & Not bv \\
\hline Multimodal MRI quantification of the common neurostructural bases within the FTD-ALS continuum. & Data Not Reported \\
\hline $\begin{array}{l}\text { Multiparametric computer-aided differential diagnosis of Alzheimer's disease and frontotemporal } \\
\text { dementia using structural and advanced MRI. }\end{array}$ & Not bv \\
\hline $\begin{array}{l}\text { Multiparametric MRI to distinguish early onset Alzheimer's disease and behavioural variant of } \\
\text { frontotemporal dementia. }\end{array}$ & Not Case Control \\
\hline Music recognition in frontotemporal lobar degeneration and Alzheimer disease. & Data Not Reported \\
\hline $\begin{array}{l}\text { My belief or yours? Differential theory of mind deficits in frontotemporal dementia and Alzheimer's } \\
\text { disease. }\end{array}$ & Data Not Reported \\
\hline Naming and conceptual understanding in frontotemporal dementia & Not Case Control \\
\hline Network degeneration and dysfunction in presymptomatic C9ORF72 expansion carriers. & Irrelevant \\
\hline
\end{tabular}




\begin{tabular}{|c|c|}
\hline $\begin{array}{l}\text { Network-selective vulnerability of the human cerebellum to Alzheimer's disease and frontotemporal } \\
\text { dementia. }\end{array}$ & ROI \\
\hline Neural basis of interpersonal traits in neurodegenerative diseases. & Data Not Reported \\
\hline Neural correlates of activities of daily living in frontotemporal dementia. & Not bv \\
\hline $\begin{array}{l}\text { Neural correlates of behavioural symptoms in behavioural variant frontotemporal dementia and } \\
\text { Alzheimer's disease: Employment of a visual MRI rating scale. }\end{array}$ & Irrelevant \\
\hline Neural correlates of empathic impairment in the behavioral variant of frontotemporal dementia. & Data Not Reported \\
\hline Neural correlates of episodic memory in behavioral variant frontotemporal dementia. & $\begin{array}{l}\text { No voxel-Based Morphometry } \\
\text { Was Performed on MRI Images. }\end{array}$ \\
\hline $\begin{array}{l}\text { Neural correlates of reduced awareness in instrumental activities of daily living in frontotemporal } \\
\text { dementia. }\end{array}$ & Data Not Reported \\
\hline $\begin{array}{l}\text { Neural substrates of episodic memory dysfunction in behavioural variant frontotemporal dementia with } \\
\text { and without C9ORF72 expansions }\end{array}$ & Data Not Reported \\
\hline Neural substrates of socioemotional self-awareness in neurodegenerative disease & Data Not Reported \\
\hline $\begin{array}{l}\text { Neuroanatomical correlates of emotional blunting in behavioral variant frontotemporal dementia and } \\
\text { early-onset Alzheimer's disease. }\end{array}$ & $\begin{array}{l}\text { Tensor-based Morphometry } \\
\text { (TBM) }\end{array}$ \\
\hline Neuroanatomical profiles of personality change in frontotemporal lobar degeneration. & Data Not Reported \\
\hline Neuroanatomy of apathy and disinhibition in frontotemporal lobar degeneration. & Not bv \\
\hline Neuroanatomy of the self: evidence from patients with frontotemporal dementia. & Not Case Control \\
\hline Neurodegeneration Behind Bars: From molecules to jurisprudence & Review Article \\
\hline $\begin{array}{l}\text { Neuroimaging of Inflammation in Memory and Related Other Disorders (NIMROD) study protocol: a } \\
\text { deep phenotyping cohort study of the role of brain inflammation in dementia, depression and other } \\
\text { neurological illnesses. }\end{array}$ & Irrelevant \\
\hline Neuroimaging patterns along the ALS-FTD spectrum: a multiparametric imaging study. & Data Not Reported \\
\hline $\begin{array}{l}\text { Neuroimaging signatures of frontotemporal dementia genetics: C9ORF72, tau, progranulin and } \\
\text { sporadics. }\end{array}$ & Data Not Reported \\
\hline Neuroinflammation and protein aggregation co-localize across the frontotemporal dementia spectrum & 11C-PK-11195 PET scanning \\
\hline $\begin{array}{l}\text { Neuropsychiatric effects of neurodegeneration of the medial versus lateral ventral prefrontal cortex in } \\
\text { humans. }\end{array}$ & Not bv \\
\hline $\begin{array}{l}\text { Neuropsychological and Neuroanatomical Correlates of the Social Norms Questionnaire in } \\
\text { Frontotemporal Dementia Versus Alzheimer's Disease. }\end{array}$ & Not Case Control \\
\hline Niemann-Pick Disease Type C Misdiagnosed as Cerebral Palsy: A Case Report & Case Report \\
\hline Novel cerebrospinal fluid biomarkers of axonal degeneration in frontotemporal dementia. & Not bv \\
\hline $\begin{array}{l}\text { Occupational attainment influences longitudinal decline in behavioral variant frontotemporal } \\
\text { degeneration }\end{array}$ & Data Not Reported \\
\hline Olfactory function in corticobasal syndrome and frontotemporal dementia. & ROI \\
\hline Open-label study of the short-term effects of memantine on FDG-PET in frontotemporal dementia. & Data Not Reported \\
\hline
\end{tabular}


Overlap in frontotemporal atrophy between normal aging and patients with frontotemporal dementias. Pain and temperature processing in dementia: a clinical and neuroanatomical analysis.

Pain Anticipation and Nocebo-Related Responses: A Descriptive Mini-Review of Functional Neuroimaging Studies in Normal Subjects and Precious Hints on Pain Processing in the Context of Neurodegenerative Disorders

Pathophysiology of the behavioral variant of frontotemporal lobar degeneration: A study combining MRI and FDG-PET

Pattern of glucose hypometabolism in frontotemporal dementia with motor neuron disease.

Patterns of atrophy in pathologically confirmed dementias: a voxelwise analysis.

Patterns of atrophy in pathologically confirmed FTLD with and without motor neuron degeneration.

Patterns of brain atrophy in clinical variants of frontotemporal lobar degeneration.

Patterns of brain atrophy in frontotemporal dementia and semantic dementia.

Patterns of cortical thinning in Alzheimer's disease and frontotemporal dementia.

Patterns of frontal lobe atrophy in frontotemporal dementia: a volumetric MRI study.

Patterns of gray matter atrophy in genetic frontotemporal dementia: results from the GENFI study.

Patterns of MRI atrophy in tau positive and ubiquitin positive frontotemporal lobar degeneration.

Patterns of regional cerebellar atrophy in genetic frontotemporal dementia.

Patterns of striatal degeneration in frontotemporal dementia.

Patterns of white matter atrophy in frontotemporal lobar degeneration.

Phenotype variability in progranulin mutation carriers: a clinical, neuropsychological, imaging and genetic study.

Pittsburgh compound B imaging and cerebrospinal fluid amyloid- $\hat{\mathrm{I}}^{2}$ in a multicentre European memory clinic study.

Posterior cerebral atrophy in the absence of medial temporal lobe atrophy in pathologically-confirmed

Alzheimer's disease.

Predominant subcortical accumulation of (18)F-flortaucipir binding in behavioral variant frontotemporal dementia.

Profiles of white matter tract pathology in frontotemporal dementia.

Prognosis of Patients with Behavioral Variant Frontotemporal Dementia Who have Focal Versus Diffuse

Frontal Atrophy.

Progression of logopenic aphasia to frontotemporal dementia in an amyloid $\dot{\zeta}^{2}$-negative and (18) F-THK5351-positive patient

Progressive brainstem pathology in motor neuron diseases: Imaging data from amyotrophic lateral sclerosis and primary lateral sclerosis

Prominent auditory deficits in primary progressive aphasia: A case study

Prosocial deficits in behavioral variant frontotemporal dementia relate to reward network atrophy.

Putaminal volume in frontotemporal lobar degeneration and Alzheimer disease: differential volumes in dementia subtypes and controls.

Qualitative Assessment of Longitudinal Changes in Phenocopy Frontotemporal Dementia

Quantifying change in individual subjects affected by frontotemporal lobar degeneration using automated longitudinal MRI volumetry.

Quantitative analysis of grey matter degeneration in FTD patients using fractal dimension analysis.

Quantitative validation of a visual rating scale for frontal atrophy: associations with clinical status,

APOE e4, CSF biomarkers and cognition.

Rates of brain atrophy over time in autopsy-proven frontotemporal dementia and Alzheimer disease.
ROI

Not bv

Review Article

ROI

Not bv

Irrelevant

Not bv

Not Case Control

Data Not Reported

No voxel-Based Morphometry

Was Performed on MRI Images.

ROI

Not bv

Not bv

Not bv

ROI

No voxel-Based Morphometry

Was Performed on MRI Images.

Irrelevant

11C-PiB PET Imaging

Not by

ROI

Data Not Reported

Irrelevant

Case Report

Irrelevant

Case Report

Data Not Reported

ROI

Not bv

ROI

No voxel-Based Morphometry

Was Performed on MRI Images.

Irrelevant

Not bv 


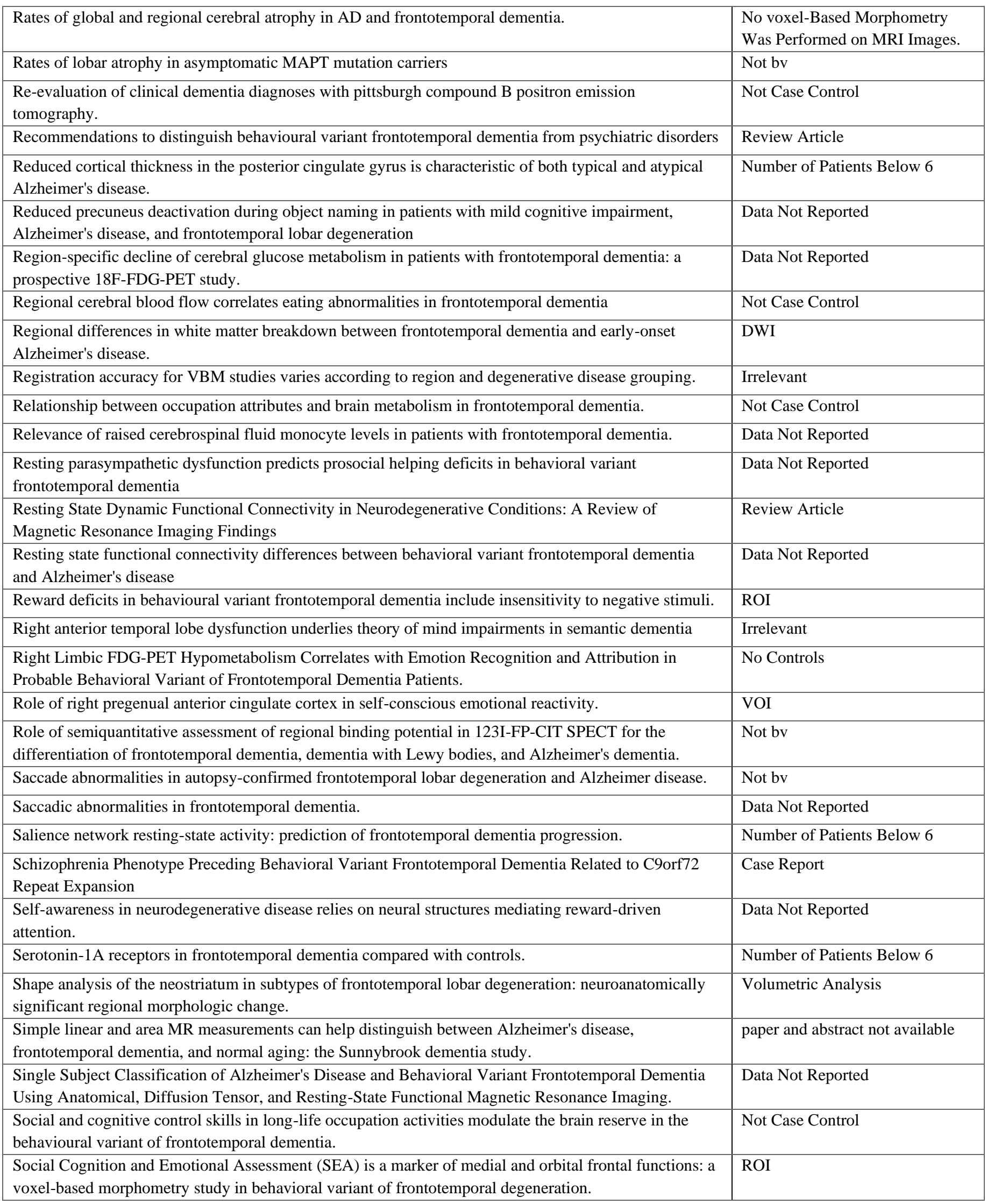




\begin{tabular}{|c|c|}
\hline $\begin{array}{l}\text { Social cognition, executive functioning, and neuroimaging correlates of empathic deficits in } \\
\text { frontotemporal dementia. }\end{array}$ & Data Not Reported \\
\hline $\begin{array}{l}\text { Social conceptual impairments in frontotemporal lobar degeneration with right anterior temporal } \\
\text { hypometabolism. }\end{array}$ & Not bv \\
\hline Social mind representation: where does it fail in frontotemporal dementia? & Data Not Reported \\
\hline $\begin{array}{l}\text { Spatiotemporal analysis for detection of pre-symptomatic shape changes in neurodegenerative diseases: } \\
\text { Initial application to the GENFI cohort }\end{array}$ & Not bv \\
\hline $\begin{array}{l}\text { Striatal Atrophy in the Behavioural Variant of Frontotemporal Dementia: Correlation with Diagnosis, } \\
\text { Negative Symptoms and Disease Severity. }\end{array}$ & ROI \\
\hline Striatal dopamine transporter and extrapyramidal symptoms in frontotemporal dementia. & ROI \\
\hline Structural anatomy of empathy in neurodegenerative disease. & Data Not Reported \\
\hline $\begin{array}{l}\text { Structural and functional brain abnormalities place phenocopy frontotemporal dementia (FTD) in the } \\
\text { FTD spectrum. }\end{array}$ & ROI \\
\hline $\begin{array}{l}\text { Structural MRI in frontotemporal dementia: comparisons between hippocampal volumetry, tensor-based } \\
\text { morphometry and voxel-based morphometry. }\end{array}$ & ROI \\
\hline Structural neuroanatomy of face processing in frontotemporal lobar degeneration. & Data Not Reported \\
\hline $\begin{array}{l}\text { Structural neuroimaging of social cognition in progressive non-fluent aphasia and behavioral variant of } \\
\text { frontotemporal dementia. }\end{array}$ & Not bv \\
\hline Subacute combined degeneration of the spinal cord masking motor neuron disease: a case report & Case Report \\
\hline Subcortical and Deep Cortical Atrophy in Frontotemporal Dementia due to Granulin Mutations. & Not bv \\
\hline $\begin{array}{l}\text { Subcortical atrophy in frontotemporal dementia and Alzheimer's disease: Significance for the differential } \\
\text { diagnosis and correlations with clinical manifestations. }\end{array}$ & $\begin{array}{l}\text { No voxel-Based Morphometry } \\
\text { Was Performed on MRI Images. }\end{array}$ \\
\hline $\begin{array}{l}\text { Survival in frontotemporal lobar degeneration and related disorders: Latent class predictors and brain } \\
\text { functional correlates }\end{array}$ & Irrelevant \\
\hline $\begin{array}{l}\text { Tackling variability: A multicenter study to provide a gold-standard network approach for } \\
\text { frontotemporal dementia. }\end{array}$ & Connectivity Matrices \\
\hline Tau forms in CSF as a reliable biomarker for progressive supranuclear palsy. & Irrelevant \\
\hline $\begin{array}{l}\text { Tau haplotype influences cerebral perfusion pattern in frontotemporal lobar degeneration and related } \\
\text { disorders. }\end{array}$ & 99mTc-ECD SPECT acquisition \\
\hline TDP-43 subtypes are associated with distinct atrophy patterns in frontotemporal dementia. & Not bv \\
\hline Temporal lobe rating scale: application to Alzheimer's disease and frontotemporal dementia. & ROI \\
\hline Temporal variant of frontotemporal dementia in C9orf72 repeat expansion carriers: two case studies & Case Series \\
\hline Temporoparietal atrophy: a marker of AD pathology independent of clinical diagnosis. & Not bv \\
\hline $\begin{array}{l}\text { Temporoparietal hypometabolism in frontotemporal lobar degeneration and associated imaging } \\
\text { diagnostic errors. }\end{array}$ & Not bv \\
\hline $\begin{array}{l}\text { Thalamic nuclei in frontotemporal dementia: Mediodorsal nucleus involvement is universal but pulvinar } \\
\text { atrophy is unique to C9orf } 72\end{array}$ & ROI \\
\hline $\begin{array}{l}\text { The 5-HTTLPR variant in the serotonin transporter gene modifies degeneration of brain regions } \\
\text { important for emotion in behavioral variant frontotemporal dementia. }\end{array}$ & Data Not Reported \\
\hline $\begin{array}{l}\text { The added value of } 18 \text {-fluorodeoxyglucose-positron emission tomography in the diagnosis of the } \\
\text { behavioral variant of frontotemporal dementia. }\end{array}$ & Irrelevant \\
\hline $\begin{array}{l}\text { The Association Between Distinct Frontal Brain Volumes and Behavioral Symptoms in Mild Cognitive } \\
\text { Impairment, Alzheimer's Disease, and Frontotemporal Dementia }\end{array}$ & ROI \\
\hline The attribution of animacy and agency in frontotemporal dementia versus Alzheimer's disease. & ROI \\
\hline $\begin{array}{l}\text { The behavioural/dysexecutive variant of Alzheimer's disease: clinical, neuroimaging and pathological } \\
\text { features. }\end{array}$ & Irrelevant \\
\hline The brain basis of musicophilia: evidence from frontotemporal lobar degeneration. & Not bv \\
\hline The Cerebellum in Frontotemporal Dementia: a Meta-Analysis of Neuroimaging Studies & Meta-Analysis \\
\hline The clinical and anatomical heterogeneity of environmental dependency phenomena. & Data Not Reported \\
\hline
\end{tabular}




\begin{tabular}{|c|c|}
\hline $\begin{array}{l}\text { The correlation of cognitive decline with frontotemporal dementia induced annualized gray matter loss } \\
\text { using diffeomorphic morphometry. }\end{array}$ & Not bv \\
\hline $\begin{array}{l}\text { The Evolution of Caregiver Burden in Â Frontotemporal Dementia with and without Amyotrophic } \\
\text { Lateral Sclerosis. }\end{array}$ & Not Case Control \\
\hline $\begin{array}{l}\text { The frontal-anatomic specificity of design fluency repetitions and their diagnostic relevance for } \\
\text { behavioral variant frontotemporal dementia. }\end{array}$ & Irrelevant \\
\hline $\begin{array}{l}\text { The inner fluctuations of the brain in presymptomatic Frontotemporal Dementia: The chronnectome } \\
\text { fingerprint }\end{array}$ & Not bv \\
\hline The Italian Alzheimer's Disease Neuroimaging Initiative (I-ADNI): validation of structural MR imaging. & Irrelevant \\
\hline The Language Profile of Behavioral Variant Frontotemporal Dementia. & Data Not Reported \\
\hline $\begin{array}{l}\text { The longitudinal decline of white matter microstructural integrity in behavioral variant frontotemporal } \\
\text { dementia and its association with executive function }\end{array}$ & DWI \\
\hline $\begin{array}{l}\text { The longitudinal evaluation of familial frontotemporal dementia subjects protocol: Framework and } \\
\text { methodology }\end{array}$ & Protocol Article \\
\hline The neural basis for establishing a focal point in pure coordination games. & Volumetric MRI \\
\hline $\begin{array}{l}\text { The neural correlates and clinical characteristics of psychosis in the frontotemporal dementia continuum } \\
\text { and the C9orf72 expansion. }\end{array}$ & Data Not Reported \\
\hline The orbitofrontal cortex is involved in emotional enhancement of memory: evidence from the dementias. & ROI \\
\hline The Phenotype of the C9ORF72 Expansion Carriers According to Revised Criteria for bvFTD. & Not Case Control \\
\hline The power of neuroimaging biomarkers for screening frontotemporal dementia. & Volumetric MRI and DWI \\
\hline The role of MAPT gene in Chinese dementia patients: A P3011 pedigree study and brief literature review & No Control \\
\hline $\begin{array}{l}\text { The structural neuroanatomy of music emotion recognition: evidence from frontotemporal lobar } \\
\text { degeneration. }\end{array}$ & Data Not Reported \\
\hline The tauopathy associated with mutation +3 in intron 10 of Tau: characterization of the MSTD family. & Not bv \\
\hline The underlying mechanisms of verbal fluency deficit in frontotemporal dementia and semantic dementia. & Data Not Reported \\
\hline $\begin{array}{l}\text { The Use of Biomarkers and Genetic Screening to Diagnose Frontotemporal Dementia: Evidence and } \\
\text { Clinical Implications }\end{array}$ & Review Article \\
\hline $\begin{array}{l}\text { The value of FDG-PET/CT by using 3-dimensional stereotactic surface projection software analysis in } \\
\text { the differential diagnosis of dementia. }\end{array}$ & Not bv \\
\hline $\begin{array}{l}\text { Theory of mind impairment in patients with behavioural variant fronto-temporal dementia (bv-FTD) } \\
\text { increases caregiver burden. }\end{array}$ & Data Not Reported \\
\hline Topographical patterns of lobar atrophy in frontotemporal dementia and Alzheimer's disease. & Not bv \\
\hline $\begin{array}{l}\text { Topography of primitive reflexes in dementia: an F-18 fluorodeoxyglucose positron emission } \\
\text { tomography study. }\end{array}$ & Not bv \\
\hline Trajectories of brain and hippocampal atrophy in FTD with mutations in MAPT or GRN. & Not bv \\
\hline $\begin{array}{l}\text { Trajectory of lobar atrophy in asymptomatic and symptomatic GRN mutation carriers: a longitudinal } \\
\text { MRI study }\end{array}$ & Not bv \\
\hline Two distinct subtypes of right temporal variant frontotemporal dementia. & Not bv \\
\hline Typical cerebral metabolic patterns in neurodegenerative brain diseases. & Not bv \\
\hline Unified voxel- and tensor-based morphometry (UVTBM) using registration confidence. & Not bv \\
\hline Urinary incontinence and its functional anatomy in frontotemporal lobar degenerations. & Not bv \\
\hline $\begin{array}{l}\text { Usefulness of measurement of the temporal stem on magnetic resonance imaging in the diagnosis of } \\
\text { frontotemporal dementia. }\end{array}$ & Not bv \\
\hline $\begin{array}{l}\text { Using simultaneous PET/MRI to compare the accuracy of diagnosing frontotemporal dementia by } \\
\text { arterial spin labelling MRI and FDG-PET. }\end{array}$ & Not bv \\
\hline Validating FDG-PET as a biomarker for frontotemporal dementia. & Irrelevant \\
\hline Validation of consensus panel diagnosis in dementia. & Irrelevant \\
\hline Validation of the Spanish version of the Addenbrooke's Cognitive Examination in a rural community in & Irrelevant \\
\hline
\end{tabular}




\begin{tabular}{|c|c|}
\hline Spain. & \\
\hline $\begin{array}{l}\text { Value of neuropsychological testing, imaging, and CSF biomarkers for the differential diagnosis and } \\
\text { prognosis of clinically ambiguous dementia. }\end{array}$ & Irrelevant \\
\hline $\begin{array}{l}\text { Variant-specific vulnerability in metabolic connectivity and resting-state networks in behavioural variant } \\
\text { of frontotemporal dementia }\end{array}$ & Data Not Reported \\
\hline VBM signatures of abnormal eating behaviours in frontotemporal lobar degeneration. & Not bv \\
\hline Ventricular volume expansion in presymptomatic genetic frontotemporal dementia & Not bv \\
\hline $\begin{array}{l}\text { Ventromedial-frontopolar prefrontal cortex atrophy correlates with insight loss in frontotemporal } \\
\text { dementia and Alzheimer's disease. }\end{array}$ & Not Case Control \\
\hline $\begin{array}{l}\text { Visual assessment of atrophy on magnetic resonance imaging in the diagnosis of pathologically } \\
\text { confirmed young-onset dementias. }\end{array}$ & Irrelevant \\
\hline Visual perceptual organization deficits in Alzheimer's dementia. & Number of Patients Below 6 \\
\hline $\begin{array}{l}\text { Visual rating and volumetry of the medial temporal lobe on magnetic resonance imaging in dementia: a } \\
\text { comparative study. }\end{array}$ & Irrelevant \\
\hline Visual search patterns in semantic dementia show paradoxical facilitation of binding processes. & ROI \\
\hline Visuospatial dysfunction in Alzheimer's disease and behavioural variant frontotemporal dementia & 11C-PiB PET Imaging \\
\hline Volumetric study of lobar atrophy in Pick complex and Alzheimer's disease. & $\begin{array}{l}\text { No voxel-Based Morphometry } \\
\text { Was Performed on MRI Images. }\end{array}$ \\
\hline Voxel-based classification of FDG PET in dementia using inter-scanner normalization. & Irrelevant \\
\hline $\begin{array}{l}\text { Voxel-based morphometry in frontotemporal lobar degeneration with ubiquitin-positive inclusions with } \\
\text { and without progranulin mutations. }\end{array}$ & Not bv \\
\hline $\begin{array}{l}\text { Voxel-based morphometry in patients with obsessive-compulsive behaviors in behavioral variant } \\
\text { frontotemporal dementia. }\end{array}$ & Data Not Reported \\
\hline Voxel-based morphometry in tau-positive and tau-negative frontotemporal lobar degenerations. & Not bv \\
\hline Voxel-based morphometry patterns of atrophy in FTLD with mutations in MAPT or PGRN & Not bv \\
\hline When one loses empathy: its effect on carers of patients with dementia. & Irrelevant \\
\hline $\begin{array}{l}\text { White Matter Changes Associated with Resting Sympathetic Tone in Frontotemporal Dementia vs. } \\
\text { Alzheimer's Disease. }\end{array}$ & $\begin{array}{l}\text { Cortical volumes calculated by } \\
\text { FreeSurfer and DWI }\end{array}$ \\
\hline White matter damage in frontotemporal dementia and Alzheimer's disease measured by diffusion MRI. & DWI \\
\hline White matter damage in frontotemporal lobar degeneration spectrum. & ROI \\
\hline $\begin{array}{l}\text { White matter disease contributes to apathy and disinhibition in behavioral variant frontotemporal } \\
\text { dementia. }\end{array}$ & DWI \\
\hline White matter tract signatures of impaired social cognition in frontotemporal lobar degeneration. & DWI \\
\hline Why are patients with progressive nonfluent aphasia nonfluent? & $\begin{array}{l}\text { No voxel-Based Morphometry } \\
\text { Was Performed on MRI Images. }\end{array}$ \\
\hline
\end{tabular}




\section{Supplementary references}

1. $\quad$ Lee, S.E., et al., Altered network connectivity in frontotemporal dementia with C9orf72 hexanucleotide repeat expansion. Brain, 2014. 137(Pt 11): p. 3047-60.

2. $\quad$ Seeley, W.W., et al., Frontal paralimbic network atrophy in very mild behavioral variant frontotemporal dementia. Arch Neurol, 2008. 65(2): p. 249-55.

3. Mandelli, M.L., et al., Two insular regions are differentially involved in behavioral variant FTD and nonfluent/agrammatic variant PPA. Cortex, 2016. 74: p. 149-57.

4. Agosta, F., et al., Apolipoprotein E epsilon4 is associated with disease-specific effects on brain atrophy in Alzheimer's disease and frontotemporal dementia. Proc Natl Acad Sci U S A, 2009. 106(6): p. 2018-22.

5. $\quad$ Chiong, W., et al., The salience network causally influences default mode network activity during moral reasoning. Brain, 2013. 136(Pt 6): p. 1929-41.

6. Lagarde, J., et al., Are frontal cognitive and atrophy patterns different in PSP and bvFTD? A comparative neuropsychological and VBM study. PLoS One, 2013. 8(11): p. e80353.

7. Lagarde, J., et al., Why do patients with neurodegenerative frontal syndrome fail to answer: 'In what way are an orange and a banana alike?'. Brain, 2015. 138(Pt 2): p. 456-71.

8. Kumfor, F., et al., Beyond the face: how context modulates emotion processing in frontotemporal dementia subtypes. Brain, 2018. 141(4): p. 1172-1185.

9. $\quad$ Kamminga, J., et al., Differentiating between right-lateralised semantic dementia and behavioural-variant frontotemporal dementia: an examination of clinical characteristics and emotion processing. J Neurol Neurosurg Psychiatry, 2015. 86(10): p. 1082-8.

10. Kumfor, F., et al., Frontal and temporal lobe contributions to emotional enhancement of memory in behavioral-variant frontotemporal dementia and Alzheimer's disease. Front Behav Neurosci, 2014. 8: p. 225.

11. Kumfor, F., et al., Do I know you? Examining face and object memory in frontotemporal dementia. Neuropsychologia, 2015. 71: p. 101-11.

12. Kumfor, F., et al., Apathy in Alzheimer's disease and frontotemporal dementia: Distinct clinical profiles and neural correlates. Cortex, 2018. 103: p. 350-359.

13. Kumfor, F., et al., Assessing the "social brain" in dementia: Applying TASIT-S. Cortex, 2017. 93: p. 166-177.

14. Hutchings, R., et al., Why Should I Care? Dimensions of Socio-Emotional Cognition in YoungerOnset Dementia. J Alzheimers Dis, 2015. 48(1): p. 135-47.

15. Kumfor, F., et al., Discrete Neural Correlates for the Recognition of Negative Emotions: Insights from Frontotemporal Dementia. PLoS One, 2013. 8(6): p. e67457.

16. Ahmed, R.M., et al., Assessment of Eating Behavior Disturbance and Associated Neural Networks in Frontotemporal Dementia. JAMA Neurol, 2016. 73(3): p. 282-90.

17. Devenney, E., et al., Progression in Behavioral Variant Frontotemporal Dementia: A Longitudinal Study. JAMA Neurol, 2015. 72(12): p. 1501-9.

18. Ahmed, R.M., et al., Neural networks associated with body composition in frontotemporal dementia. Ann Clin Transl Neurol, 2019. 6(9): p. 1707-1717.

19. Donnelly-Kehoe, P.A., et al., Robust automated computational approach for classifying frontotemporal neurodegeneration: Multimodal/multicenter neuroimaging. Alzheimers Dement (Amst), 2019. 11: p. 588-598.

20. Kumfor, F., et al., Facial expressiveness and physiological arousal in frontotemporal dementia: Phenotypic clinical profiles and neural correlates. Cogn Affect Behav Neurosci, 2019. 19(1): p. 197-210.

21. Wei, G., et al., Disease-specific profiles of apathy in Alzheimer's disease and behavioural-variant frontotemporal dementia differ across the disease course. J Neurol, 2020. 267(4): p. 1086-1096. 
22. Wilson, N.A., et al., Scene construction impairments in frontotemporal dementia: Evidence for a primary hippocampal contribution. Neuropsychologia, 2020. 137: p. 107327.

23. Irish, M., J.R. Hodges, and O. Piguet, Episodic future thinking is impaired in the behavioural variant of frontotemporal dementia. Cortex, 2013. 49(9): p. 2377-88.

24. Irish, M., et al., Common and unique gray matter correlates of episodic memory dysfunction in frontotemporal dementia and Alzheimer's disease. Hum Brain Mapp, 2014. 35(4): p. 1422-35.

25. Irish, M., et al., Grey and white matter correlates of recent and remote autobiographical memory retrieval--insights from the dementias. PLoS One, 2014. 9(11): p. e113081.

26. Irish, M., et al., Neural Substrates of Semantic Prospection - Evidence from the Dementias. Front Behav Neurosci, 2016. 10: p. 96.

27. Dermody, N., et al., Prospective Memory Impairments in Alzheimer's Disease and Behavioral Variant Frontotemporal Dementia: Clinical and Neural Correlates. J Alzheimers Dis, 2016. 50(2): p. 425-41.

28. Dermody, N., et al., Uncovering the Neural Bases of Cognitive and Affective Empathy Deficits in Alzheimer's Disease and the Behavioral-Variant of Frontotemporal Dementia. J Alzheimers Dis, 2016. 53(3): p. 801-16.

29. Hornberger, M., J. Geng, and J.R. Hodges, Convergent grey and white matter evidence of orbitofrontal cortex changes related to disinhibition in behavioural variant frontotemporal dementia. Brain, 2011. 134(Pt 9): p. 2502-12.

30. Chen, Y., et al., Cerebellar atrophy and its contribution to cognition in frontotemporal dementias. Ann Neurol, 2018. 84(1): p. 98-109.

31. Irish, M., J.R. Hodges, and O. Piguet, Right anterior temporal lobe dysfunction underlies theory of mind impairments in semantic dementia. Brain, 2014. 137(Pt 4): p. 1241-53.

32. O'Callaghan, C., et al., Hippocampal atrophy and intrinsic brain network dysfunction relate to alterations in mind wandering in neurodegeneration. Proc Natl Acad Sci U S A, 2019. 116(8): p. 3316-3321.

33. Tu, S., et al., Lost in spatial translation - A novel tool to objectively assess spatial disorientation in Alzheimer's disease and frontotemporal dementia. Cortex, 2015. 67: p. 83-94.

34. Baez, S., et al., Brain structural correlates of executive and social cognition profiles in behavioral variant frontotemporal dementia and elderly bipolar disorder. Neuropsychologia, 2019. 126: p. 159-169.

35. Baez, S., et al., Orbitofrontal and limbic signatures of empathic concern and intentional harm in the behavioral variant frontotemporal dementia. Cortex, 2016. 75: p. 20-32.

36. Baez, S., et al., Integration of Intention and Outcome for Moral Judgment in Frontotemporal Dementia: Brain Structural Signatures. Neurodegener Dis, 2016. 16(3-4): p. 206-17.

37. Cousins, K.A., et al., Cognitive and anatomic double dissociation in the representation of concrete and abstract words in semantic variant and behavioral variant frontotemporal degeneration. Neuropsychologia, 2016. 84: p. 244-51.

38. McMillan, C.T., et al., Converging evidence for the processing costs associated with ambiguous quantifier comprehension. Front Psychol, 2013. 4: p. 153.

39. Grossman, M., et al., What's in a name: voxel-based morphometric analyses of MRI and naming difficulty in Alzheimer's disease, frontotemporal dementia and corticobasal degeneration. Brain, 2004. 127(Pt 3): p. 628-49.

40. Morgan, B., et al., Some is not enough: quantifier comprehension in corticobasal syndrome and behavioral variant frontotemporal dementia. Neuropsychologia, 2011. 49(13): p. 3532-41.

41. Libon, D.J., et al., Neurocognitive contributions to verbal fluency deficits in frontotemporal lobar degeneration. Neurology, 2009. 73(7): p. 535-42.

42. Nevler, N., et al., Automatic measurement of prosody in behavioral variant FTD. Neurology, 2017. 89(7): p. 650-656.

43. Massimo, L., et al., Self-appraisal in behavioural variant frontotemporal degeneration. J Neurol Neurosurg Psychiatry, 2013. 84(2): p. 148-53. 
44. Ash, S., et al., Dissociation of quantifiers and object nouns in speech in focal neurodegenerative disease. Neuropsychologia, 2016. 89: p. 141-152.

45. Healey, M.L., et al., Getting on the same page: the neural basis for social coordination deficits in behavioral variant frontotemporal degeneration. Neuropsychologia, 2015. 69: p. 56-66.

46. Kanda, T., et al., Comparison of grey matter and metabolic reductions in frontotemporal dementia using FDG-PET and voxel-based morphometric MR studies. Eur J Nucl Med Mol Imaging, 2008. 35(12): p. 2227-34.

47. Franceschi, M., et al., Glucose metabolism and serotonin receptors in the frontotemporal lobe degeneration. Ann Neurol, 2005. 57(2): p. 216-25.

48. Salmon, E., et al., Predominant ventromedial frontopolar metabolic impairment in frontotemporal dementia. Neuroimage, 2003. 20(1): p. 435-40.

49. Tuovinen, T., et al., The Effect of Gray Matter ICA and Coefficient of Variation Mapping of BOLD Data on the Detection of Functional Connectivity Changes in Alzheimer's Disease and bvFTD. Front Hum Neurosci, 2016. 10: p. 680.

50. Rytty, R., et al., GroupICA dual regression analysis of resting state networks in a behavioral variant of frontotemporal dementia. Front Hum Neurosci, 2013. 7: p. 461.

51. Melloni, M., et al., Your perspective and my benefit: multiple lesion models of self-other integration strategies during social bargaining. Brain, 2016. 139(11): p. 3022-3040.

52. Fernández-Matarrubia, M., et al., Episodic Memory Dysfunction in Behavioral Variant Frontotemporal Dementia: A Clinical And FDG-PET Study. J Alzheimers Dis, 2017. 57(4): p. 1251-1264.

53. Matías-Guiu, J.A., et al., Inhibition impairment in frontotemporal dementia, amyotrophic lateral sclerosis, and Alzheimer's disease: clinical assessment and metabolic correlates. Brain Imaging Behav, 2019. 13(3): p. 651-659.

54. Hafkemeijer, A., et al., A Longitudinal Study on Resting State Functional Connectivity in Behavioral Variant Frontotemporal Dementia and Alzheimer's Disease. J Alzheimers Dis, 2017. 55(2): p. 521-537.

55. Farb, N.A., et al., Abnormal network connectivity in frontotemporal dementia: evidence for prefrontal isolation. Cortex, 2013. 49(7): p. 1856-73.

56. Bertoux, M., et al., Structural Anatomical Investigation of Long-Term Memory Deficit in Behavioral Frontotemporal Dementia. J Alzheimers Dis, 2018. 62(4): p. 1887-1900.

57. Santamaría-García, H., et al., First Symptoms and Neurocognitive Correlates of Behavioral Variant Frontotemporal Dementia. J Alzheimers Dis, 2016. 54(3): p. 957-970.

58. Boccardi, M., et al., Frontotemporal dementia as a neural system disease. Neurobiol Aging, 2005. 26(1): p. 37-44.

59. Jeong, Y., et al., 18F-FDG PET findings in frontotemporal dementia: an SPM analysis of 29 patients. J Nucl Med, 2005. 46(2): p. 233-9.

60. Diehl, J., et al., Cerebral metabolic patterns at early stages of frontotemporal dementia and semantic dementia. A PET study. Neurobiol Aging, 2004. 25(8): p. 1051-6.

61. Laws, S.M., et al., Association of the tau haplotype $\mathrm{H} 2$ with age at onset and functional alterations of glucose utilization in frontotemporal dementia. Am J Psychiatry, 2007. 164(10): p. 1577-84.

62. Perneczky, R., et al., Brain reserve capacity in frontotemporal dementia: a voxel-based 18F-FDG PET study. Eur J Nucl Med Mol Imaging, 2007. 34(7): p. 1082-7.

63. Grimmer, T., et al., Region-specific decline of cerebral glucose metabolism in patients with frontotemporal dementia: a prospective 18F-FDG-PET study. Dement Geriatr Cogn Disord, 2004. 18(1): p. 32-6.

64. Peters, F., et al., Orbitofrontal dysfunction related to both apathy and disinhibition in frontotemporal dementia. Dement Geriatr Cogn Disord, 2006. 21(5-6): p. 373-9. 
65. Raczka, K.A., et al., Executive and behavioral deficits share common neural substrates in frontotemporal lobar degeneration - a pilot FDG-PET study. Psychiatry Res, 2010. 182(3): p. 274-80.

66. Sheelakumari, R., et al., Neuroanatomical correlates of apathy and disinhibition in behavioural variant frontotemporal dementia. Brain Imaging Behav, 2020. 14(5): p. 2004-2011.

67. Caminiti, S.P., et al., Affective mentalizing and brain activity at rest in the behavioral variant of frontotemporal dementia. Neuroimage Clin, 2015. 9: p. 484-97.

68. Verfaillie, S.C., et al., Cerebral perfusion and glucose metabolism in Alzheimer's disease and frontotemporal dementia: two sides of the same coin? Eur Radiol, 2015. 25(10): p. 3050-9.

69. Meyer, S., et al., Predicting behavioral variant frontotemporal dementia with pattern classification in multi-center structural MRI data. Neuroimage Clin, 2017. 14: p. 656-662.

70. García-Cordero, I., et al., Stroke and Neurodegeneration Induce Different Connectivity Aberrations in the Insula. Stroke, 2015. 46(9): p. 2673-7.

71. Kipps, C.M., et al., Understanding social dysfunction in the behavioural variant of frontotemporal dementia: the role of emotion and sarcasm processing. Brain, 2009. 132( $\mathrm{Pt} 3)$ : $\mathrm{p}$. 592-603.

72. Morbelli, S., et al., Mapping brain morphological and functional conversion patterns in predementia late-onset bvFTD. Eur J Nucl Med Mol Imaging, 2016. 43(7): p. 1337-47.

73. Agustus, J.L., et al., Functional MRI of music emotion processing in frontotemporal dementia. Ann N Y Acad Sci, 2015. 1337(1): p. 232-40. 